\title{
Search for supersymmetry in events with opposite-sign dileptons and missing transverse energy using an artificial neural network
}

\author{
S. Chatrchyan et al.* \\ (CMS Collaboration)
}

(Received 5 January 2013; published 2 April 2013)

\begin{abstract}
In this paper, a search for supersymmetry (SUSY) is presented in events with two opposite-sign isolated leptons in the final state, accompanied by hadronic jets and missing transverse energy. An artificial neural network is employed to discriminate possible SUSY signals from a standard model background. The analysis uses a data sample collected with the CMS detector during the 2011 LHC run, corresponding to an integrated luminosity of $4.98 \mathrm{fb}^{-1}$ of proton-proton collisions at the center-of-mass energy of $7 \mathrm{TeV}$. Compared to other CMS analyses, this one uses relaxed criteria on missing transverse energy $\left(\mathscr{E}_{\mathrm{T}}>40 \mathrm{GeV}\right)$ and total hadronic transverse energy $\left(H_{\mathrm{T}}>120 \mathrm{GeV}\right)$, thus probing different regions of parameter space. Agreement is found between standard model expectation and observations, yielding limits in the context of the constrained minimal supersymmetric standard model and on a set of simplified models.
\end{abstract}

DOI: 10.1103/PhysRevD.87.072001

PACS numbers: 12.60.Jv, 13.85.Rm, 14.80.Ly

\section{INTRODUCTION}

One of the most natural extensions of the standard model (SM) of particle physics is supersymmetry (SUSY) [1-8]. Supersymmetry allows for gauge coupling unification at the energy of $10^{16} \mathrm{GeV}$, provides a good dark matter candidate [lightest supersymmetric particle (LSP)] [9], is a necessary component to explain quantum gravity in the framework of string theory, and automatically cancels the quadratic divergences in radiative corrections to the Higgs boson mass. For every particle in the standard model, SUSY introduces a superpartner, the "sparticle," with spin differing by $1 / 2$ unit from the SM particle. There are theoretical arguments that suggest sparticle masses could be less than $\sim 1 \mathrm{TeV}[7,8]$ making the experiments at the Large Hadron Collider (LHC) an ideal place for their discovery.

With the successful 2011 LHC run, an integrated luminosity of $4.98 \mathrm{fb}^{-1}$ in collisions at $7 \mathrm{TeV}$ center-of-mass energy has been collected with the Compact Muon Solenoid (CMS) experiment. This data set is used to search for the presence of SUSY particles in events with two opposite-sign leptons (electrons and muons) in the final state, utilizing an artificial neural network (ANN). Two opposite-sign leptons can be produced in a SUSY cascade through the decay of neutralinos and charginos. Assuming that $R$ parity is conserved [10], a stable, weakly interacting LSP exists, resulting in a missing transverse energy $\left(\mathscr{E}_{\mathrm{T}}\right)$ signature. The amount of missing transverse energy

*Full author list given at the end of the article.

Published by the American Physical Society under the terms of the Creative Commons Attribution 3.0 License. Further distribution of this work must maintain attribution to the author(s) and the published article's title, journal citation, and DOI. depends on the mass splittings among the heavier sparticles. So far, typical dilepton SUSY searches in CMS have required several jets with large transverse momentum, which correspond to large values of $H_{\mathrm{T}}$, the scalar sum over the transverse momenta of all jets satisfying the jet selection, and large missing transverse energy to discriminate a SUSY signal from the very large SM backgrounds. Compared with previous CMS searches $[11,12]$, this analysis uses relaxed criteria on missing transverse energy $\left(\not_{\mathrm{T}}>40 \mathrm{GeV}\right)$ and $H_{\mathrm{T}}\left(H_{\mathrm{T}}>120 \mathrm{GeV}\right)$. For SUSY models that yield events with large $\mathscr{E}_{\mathrm{T}}$, the ANN's performance is comparable to the data analyses using large $\mathbb{E}_{\mathrm{T}}$ and $H_{\mathrm{T}}$. Hence, for such models the additional power of a multivariate technique is not required to discriminate between new physics and the SM backgrounds. However, for SUSY models that yield low- $\mathbb{E}_{\mathrm{T}}$ or low- $H_{\mathrm{T}}$ signatures, the discriminating power of the ANN helps to suppress the large SM backgrounds.

The results are interpreted in the context of the constrained minimal supersymmetric standard model (CMSSM [13,14]), and a class of simplified model scenarios (SMS) [15,16]. For illustration purposes, the benchmark CMSSM point LM6 $\left(m_{0}=85 \mathrm{GeV}, m_{1 / 2}=\right.$ $400 \mathrm{GeV}, \tan \beta=10, A_{0}=0 \mathrm{GeV}$ ) is used throughout the paper. In the class of SMS considered, gluinos are pair produced, with one of them decaying as $\tilde{g} \rightarrow \tilde{\chi}_{2 j}^{0} j \rightarrow$ $\tilde{\chi}_{1}^{0} \ell^{+} \ell^{-} j j$ and the other as $\tilde{g} \rightarrow \tilde{\chi}_{2}^{0} j j$. Here $\tilde{\chi}_{2}^{0}$ is the second-lightest neutralino, $\tilde{X}_{1}^{0}$ is the lightest neutralino, and the LSP, and $\ell=e, \mu$, or $\tau$ with equal probability. This SMS thus always leads to a pair of opposite-sign leptons in the final state, in addition to the jets and $\ddot{E}_{\mathrm{T}}$. The SMS is fully described by the following parameters: the masses of the gluino $\left(m_{\tilde{g}}\right)$, and the LSP $\left(m_{\mathrm{LSP}}\right)$, along with the neutralino mass in the gluino decay which is set to $m_{\tilde{\chi}_{2}^{0}}=\left(m_{\tilde{g}}+m_{\mathrm{LSP}}\right) / 2$. 


\section{CMS DETECTOR}

A detailed description of the CMS Detector can be found elsewhere [17]. A right-handed coordinate system is used with the origin at the nominal interaction point. The $x$ axis points to the center of the LHC ring, the $y$ axis is vertical and points upward, and the $z$ axis points in the direction of the counterclockwise proton beam. The azimuthal angle $\phi$ is measured with respect to the $x$ axis in the $x-y$ plane and the polar angle $\theta$ is defined with respect to the $z$ axis, while the pseudorapidity is defined as $\eta=-\ln [\tan (\theta / 2)]$. The central feature of the CMS apparatus is a superconducting solenoid, of $6 \mathrm{~m}$ internal diameter, that produces a magnetic field of 3.8 T. Located within the field volume are the silicon pixel and strip tracker, and the barrel and endcap calorimeters $(|\eta|<3)$, composed of a crystal electromagnetic calorimeter (ECAL) and a brass and scintillator hadron calorimeter (HCAL). Calorimetry provides energy and direction measurements of electrons and hadronic jets. The detector is nearly Hermetic, allowing for energy balance measurements in the plane transverse to the beam directions. Outside the field volume, in the forward region $(3<|\eta|<5)$, there is an iron and quartz-fiber hadron calorimeter. The steel return yoke outside the solenoid is instrumented with gas-ionization detectors used to identify muons. The CMS experiment collects data using a twolevel trigger system, the Level-1 hardware trigger [18] and a high-level software trigger [19].

\section{DATA SAMPLES, TRIGGER, AND EVENT SELECTION}

Data events are selected using a set of dilepton triggers, which require the presence of at least two leptons, either two muons or two electrons or a muon-electron pair. In the case of the double-muon trigger, the selection is asymmetric with a transverse momentum $\left(p_{\mathrm{T}}\right)$ threshold of $13 \mathrm{GeV}$ for the leading (higher- $p_{\mathrm{T}}$ ) muon and $8 \mathrm{GeV}$ for the subleading one. In the case of the double-electron trigger, the selection is asymmetric with a threshold applied to the transverse energy of a cluster in the ECAL. The thresholds are fixed to $17 \mathrm{GeV}(8 \mathrm{GeV})$ for the leading (subleading) electron energy. For the muon-electron trigger, the threshold on the transverse momentum, $p_{\mathrm{T}}$ (transverse energy, $\left.E_{\mathrm{T}}\right)$ is $8 \mathrm{GeV}(17 \mathrm{GeV})$ for the muon (electron). For all triggers, additional identification and isolation criteria are also applied.

Muon candidates are reconstructed [20] by combining the information from the inner tracking system, the calorimeters, and the muon system. Electron candidates are reconstructed [21] by combining the information from the ECAL with the silicon tracker, using shower shape and track-ECAL-cluster matching variables in order to increase the sample purity. Jets are reconstructed using the anti- $k_{\mathrm{T}}$ clustering algorithm [22] with a distance parameter $\Delta R=\sqrt{(\Delta \phi)^{2}+(\Delta \eta)^{2}}=0.5$. The inputs to the jet clustering algorithm are the four-momentum vectors of reconstructed particles. Each such particle is reconstructed with the particle-flow technique [23] that combines information from several subdetectors. The measured jet transverse momenta are corrected with scale factors derived from simulation; to correct for any differences in the energy response between simulation and data, a residual correction factor derived from the latter is applied to jets in the data [24]. In general, $\mathbb{E}_{\mathrm{T}} \equiv-\left|\sum \vec{p}_{\mathrm{T}}\right|$, where the sum is taken over all final-state particles reconstructed in the CMS detector. The total transverse energy $\left(\sum E_{\mathrm{T}}\right)$ of the event is calculated as the scalar sum of the transverse energies of leptons and jets. The total hadronic transverse energy, $\left(H_{\mathrm{T}} \equiv\left|\sum \vec{p}_{\mathrm{T}}\right|\right)$, is computed as the scalar sum of the transverse energies of all reconstructed jets in the event satisfying the jet selection criteria described below.

Simulated $p p$ collision events are produced with the PYTHIA] 6.4.22 [25] generator [using underlying event tune $\mathrm{Z} 2$ which is identical to the $\mathrm{Z} 1$ tune [26] except that $\mathrm{Z} 2$ uses the CTEQ6L parton distribution functions (PDF) while Z1 uses CTEQ5L] for QCD, $W W, Z Z$, and $W Z$ samples. For $t \bar{t}$, Drell-Yan, and $W+$ jets samples the MADGRAPH 4.4.24 [27] generator is used. Events are then processed with a simulation of the CMS detector response based on GEANT4 [28]. Multiple proton-proton interactions are superimposed on the hard collision, and all simulated event samples are reweighted according to the distribution of the number of reconstructed primary vertices in data. Simulated events are reconstructed and analyzed in the same way as data events. Simulated event samples are used to train the ANN, to extrapolate background estimates from a background-enriched control region in data to the expected signal-enriched region, and to estimate systematic uncertainties.

Noncollision backgrounds are removed by applying quality requirements ensuring the presence of at least one reconstructed primary vertex [29]. Events are required to have at least two opposite-sign leptons, both electrons or muons, or an electron-muon pair, with $p_{\mathrm{T}}>20 \mathrm{GeV}$ and $|\eta|<2.4$, and at least two jets with $p_{\mathrm{T}}>30 \mathrm{GeV}$ and $|\eta|<2$.4. Jets are required to satisfy the quality criteria described in Ref. [30]. Leptons are required to be isolated from significant energy deposits and tracks in a cone of radius $\Delta R=0.3$ around the direction of the lepton. The relative combined isolation, defined as $I_{\text {rel }}^{\text {comb }}=$ $\left(\sum_{\text {tracks }} p_{\mathrm{T}}+\sum_{\mathrm{ECAL}} E_{\mathrm{T}}+\sum_{\mathrm{HCAL}} E_{\mathrm{T}}\right) / p_{\mathrm{T}}$, is required to be $<0.2$ for muons and $<0.08$ for electrons, with the latter criterion being more strict in order to reject jets misidentified as electrons.

\section{SIGNAL TO BACKGROUND DISCRIMINATION}

The ANN in this analysis is used to separate SUSY signals from SM background events, exploiting correlations among the discriminating variables, and thus providing improved results with respect to the use of sequential 
TABLE I. Expected number of signal and background (bkg.) events after the event selection criteria, and after the candidate event selection criteria for events in the signal region are applied. The next-leading-order (NLO) cross section is used for the CMSSM benchmark point LM6 yield determination. The data set resulting from the candidate event selection is used as input to the ANN. The uncertainties quoted are statistical only.

\begin{tabular}{lcc}
\hline \hline Sample & Event selection & Signal region \\
\hline$t \bar{t}$ & $17395 \pm 60$ & $8271 \pm 40$ \\
$Z+$ jets & $507316 \pm 1200$ & $4740 \pm 60$ \\
$W+$ jets & $21094 \pm 740$ & $416 \pm 40$ \\
$W W$ & $1204 \pm 10$ & $15 \pm 1$ \\
$W Z$ & $1750 \pm 8$ & $20 \pm 1$ \\
$Z Z$ & $1225 \pm 4$ & $13 \pm 1$ \\
QCD & $19578 \pm 7500$ & $1313 \pm 260$ \\
Total SM bkg. & $569562 \pm 7700$ & $14797 \pm 280$ \\
LM6 & $71 \pm 1$ & $54 \pm 1$ \\
\hline \hline
\end{tabular}

selections. Because of the presence of isolated leptons, the main SM background contributions to this analysis involve the production of $t \bar{t}$ and $Z+$ jets. The QCD multijet processes with two misidentified (fake) leptons, and $W+$ jets events with one misidentified lepton, can also be part of the background, but are significantly reduced by applying additional candidate event selection criteria described below. Finally, two leptons in the final state could be produced by $W W, W Z$, or $Z Z$ decays but their contributions are found in simulation to be negligible compared to the main backgrounds.

The candidate event selection criteria, which are imposed before the ANN training, are the following: events are required to have $E_{\mathrm{T}}>30 \mathrm{GeV}$, the distance $\Delta R$ between either of the two leading opposite-sign leptons and the closest jet is required to be $>0.2$, and the dilepton mass $M_{\ell \ell}$, formed from the two leading opposite-sign leptons, is required to be larger than $10 \mathrm{GeV}$. These criteria reject the vast majority of the background, while retaining most of the signal as shown in Table I for CMSSM benchmark point LM6. This greatly facilitates the ANN training and optimization by excluding a region heavily dominated by background in which few if any signal events are present. The signal region is defined by the candidate event selection criteria with an additional requirement on the ratio of the dilepton transverse energy $\sum E_{\mathrm{T}}^{\text {lepton }}$ to the total transverse energy (as defined in Sec. III) to be less than 0.4.

The ANN training samples are based on simulated events. A mixture of $t \bar{t}, Z+$ jets, $W+$ jets, and QCD simulated samples are used as the SM background. For the signal, a class of SMS scenarios [15] is used. For the ANN training, grid points close to the diagonal $\left(m_{\tilde{g}}=\right.$ $\left.m_{\mathrm{LSP}}\right)$ are used with $\left|m_{\tilde{g}}-m_{\mathrm{LSP}}\right|<400 \mathrm{GeV}$. These points are chosen since they exhibit low $\mathbb{E}_{\mathrm{T}}$ or $H_{\mathrm{T}}$ thresholds: more than $90 \%$ of the events have $\mathscr{E}_{\mathrm{T}}<200 \mathrm{GeV}$ or $H_{\mathrm{T}}<600 \mathrm{GeV}$.
TABLE II. Seven event, lepton and jet related variables used for the ANN construction. The transverse mass $M_{T}$ is defined as $\sqrt{\left(\sum E_{\mathrm{T}}\right)^{2}-\left(\sum \vec{p}_{\mathrm{T}}\right)^{2}}$, where $\sum E_{\mathrm{T}}$ and $\sum \vec{p}_{\mathrm{T}}$ represent the scalar and vector sums over the transverse momenta of all reconstructed jets and leptons.

\begin{tabular}{lcc}
\hline \hline Variable & Description & ANN weight (\%) \\
\hline$E_{\mathrm{T}}$ & Missing transverse energy & 22 \\
$M_{\ell \ell}$ & Dilepton mass & 20 \\
$\sum E_{\mathrm{T}}^{\text {lepton }}$ & Ratio of the energy of the dilepton & 18 \\
$\sum^{E_{\mathrm{T}}}$ & system to total transverse energy \\
$N_{\text {jets }}$ & Number of jets \\
Jet2 $p_{\mathrm{T}}$ & Subleading jet $p_{\mathrm{T}}$ & 13 \\
$M_{\mathrm{T}}$ & Transverse mass & 12 \\
Jet1 $p_{\mathrm{T}}$ & Leading jet $p_{\mathrm{T}}$ & 8 \\
\hline \hline
\end{tabular}

Several topological and kinematical variables are considered according to their potential to discriminate SM backgrounds from possible SUSY signals, taking into account the correlations among them. The variables studied are based on the general production and decay characteristics of many supersymmetric processes and are not tuned to a specific model.

Using different combinations of candidate input variables, several ANNs are constructed and compared in order to select the optimal configuration. The differences in performance are studied and quantified in terms of the signal selection efficiency as a function of background rejection. A network with seven input variables, those with the smallest degree of correlation among themselves and with the highest discriminating power, shows the best performance. The ANN variable importance is defined as sum of the weights squared of the connections between the variable's neuron in the input layer and the ones in the first hidden layer. Table II lists the seven input ANN variables along with their description, and their relative importance after the ANN training.

\section{ANN OUTPUT FOR SM BACKGROUND}

In order to quantify the level of agreement and the significance of a possible excess between data and SM expectation, it is important to provide a robust estimate of the ANN output distribution in the signal region under the SM-only hypothesis along with its systematic uncertainty.

The approach used to estimate the ANN prediction for the SM-only hypothesis from data is as follows. A signal region (SR) is defined by the set of the candidate event selection requirements and the additional criterion on the fraction of transverse energy carried by the dilepton system as described in Sec. III. A primary control region (CR) is defined by inverting two of the signal event selection criteria, the 


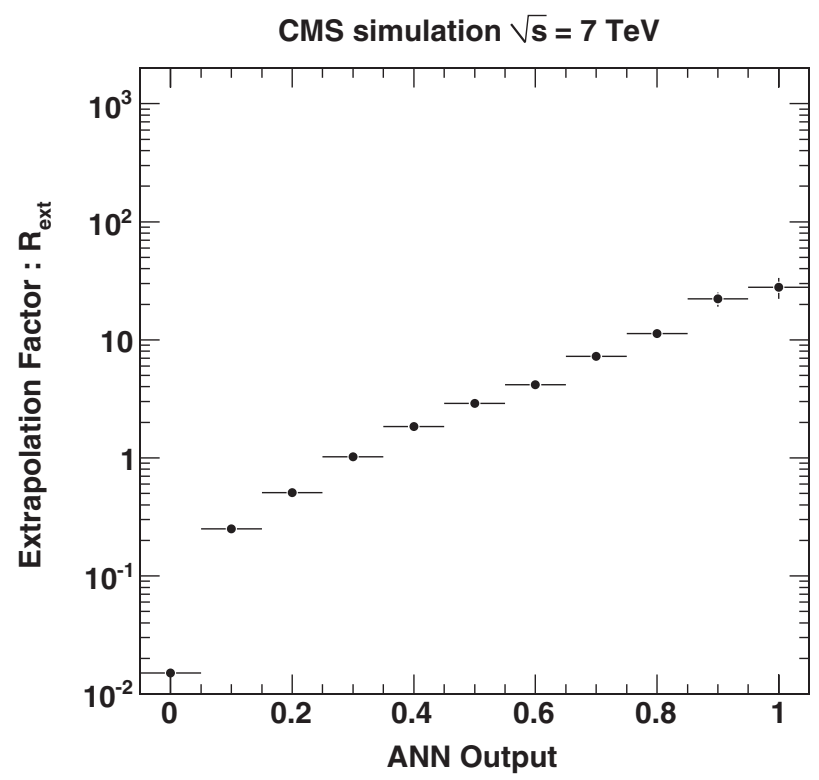

FIG. 1. Extrapolation factor $R_{\text {ext }}$, as obtained from simulated events.

total missing transverse energy and the selection cut on the fraction of transverse energy carried by the dilepton system. This region is chosen so that it is dominated by SM processes. Signal contamination in the primary control region is small: for the LM6 benchmark point it is less than $0.03 \%$, and less than $0.4 \%$ for SMS points close to the diagonal $\left(m_{\tilde{g}}=m_{\mathrm{LSP}}\right)$. The ANN output distribution in the primary control region is then obtained using data $\operatorname{ANN}(\mathrm{SM}))_{\mathrm{CR}}^{\mathrm{data}}$.

Next, an extrapolation ratio, $R_{\mathrm{Ext}}=\frac{\mathrm{ANN}(\mathrm{SM} M)_{\mathrm{SR}}^{\mathrm{MC}}}{\mathrm{ANN}(\mathrm{SM})_{\mathrm{CR}}^{\mathrm{MC}}}$ obtained from simulated events, is defined for each bin in the ANN output distribution as the ANN output for the SM-only hypothesis in the signal region divided by the ANN output for the SM-only hypothesis in the control region. The extrapolation factor, $R_{\text {ext }}$, exhibits a smooth monotonic behavior, as shown in Fig. 1.

Finally, the ANN output from data in the control region, where only SM physics is assumed to be present, is multiplied by the extrapolation factor, $R_{\text {ext }}$, to predict the ANN output $\mathrm{SM}$ in the signal region, $\mathrm{ANN}(\mathrm{SM})_{\mathrm{SR}}^{\text {prediction }}$ :

$\mathrm{ANN}(\mathrm{SM})_{\mathrm{SR}}^{\mathrm{prediction}}=\mathrm{ANN}(\mathrm{SM})_{\mathrm{CR}}^{\mathrm{data}} \times \frac{\mathrm{ANN}(\mathrm{SM})_{\mathrm{SR}}^{\mathrm{MC}}}{\mathrm{ANN}(\mathrm{SM})_{\mathrm{CR}}^{\mathrm{MC}}}$.

The primary control region is further subdivided into a $t \bar{t}$ enriched one with $\not E_{\mathrm{T}}>30 \mathrm{GeV}$ and $M_{\ell \ell} \notin[75,105] \mathrm{GeV}$, denoted as "control region A," and separately into a $Z+$ jets enriched one with $\mathbb{E}_{\mathrm{T}}>30 \mathrm{GeV}$ or $75 \mathrm{GeV}<M_{\ell \ell}<$ $105 \mathrm{GeV}$, denoted as "control region B." These are not used in the analysis. However they provide quality control cross-checks (level of agreement between data and simulation) for the two main backgrounds that affect the analysis.
Figure 2 compares the ANN output distributions of data and simulated events in the control regions as defined above. Agreement between data and simulation is observed both in the primary control region used to define the ANN output, as well as in the $t \bar{t}$ and $Z+$ jets dominated control regions $\mathrm{A}$ and $\mathrm{B}$.

Similar agreement between data and simulation for the ANN input variables in the control region is observed as well. This helps to confirm that the simulation is appropriate to train the ANN and adequate to be used for the estimation of systematic uncertainties.

\section{SYSTEMATIC UNCERTAINTIES}

Systematic uncertainties of the ANN output prediction for the SM-only hypothesis, obtained as described in Sec. V, are estimated with simulated data using the following procedure. A systematic effect is introduced into the simulated data for all events in the sample before any preselection is applied. The nominal SM extrapolation factor $R_{\text {ext }}$ is then used to obtain a new ANN output prediction for the signal region corresponding to the systematic effect under study. Next, the ANN output prediction, corresponding to the systematic alteration, is compared against the ANN output for the original sample, without any systematic effects introduced. A binned ANN output distribution is studied for this analysis. The relative difference in ANN outputs for each bin is assigned as a bin-by-bin systematic uncertainty. Similarly, the relative difference in the integrated number of events above a certain ANN output is assigned as a systematic uncertainty to the number of signal-like events. Finally, for each bin, the relative differences for all systematic effects studied are added in quadrature. This results in a bin-by-bin total systematic uncertainty in the ANN output prediction. In a similar manner the relative differences in the integrated number of events above some ANN value are added in quadrature yielding the total systematic uncertainty on the number of signal-like events.

The overall systematic uncertainties corresponding to the seven input variables used for the ANN construction, as well as the uncertainties in the cross sections of the SM backgrounds, are shown in Table III for the ANN optimal selection.

The magnitude of the systematic alterations for the jet energy scale is taken from dedicated CMS measurements [24]. While the clustered energy scale of $\ddot{E}_{\mathrm{T}}$ is known to the $3 \%$ level in CMS and the unclustered energy scale for $\not_{\mathrm{T}}$ is known to within $10 \%$ [31], this analysis uses a conservative $10 \%$ for the overall $\mathbb{E}_{\mathrm{T}}$ systematic uncertainty.

For the input ANN variables for which there is no dedicated CMS measurement, the level of agreement between data and simulation in the control region is used to obtain an estimate of the systematic uncertainty. Therefore, the control region is used to constrain the systematic uncertainties in these cases. Given the above, the difference 

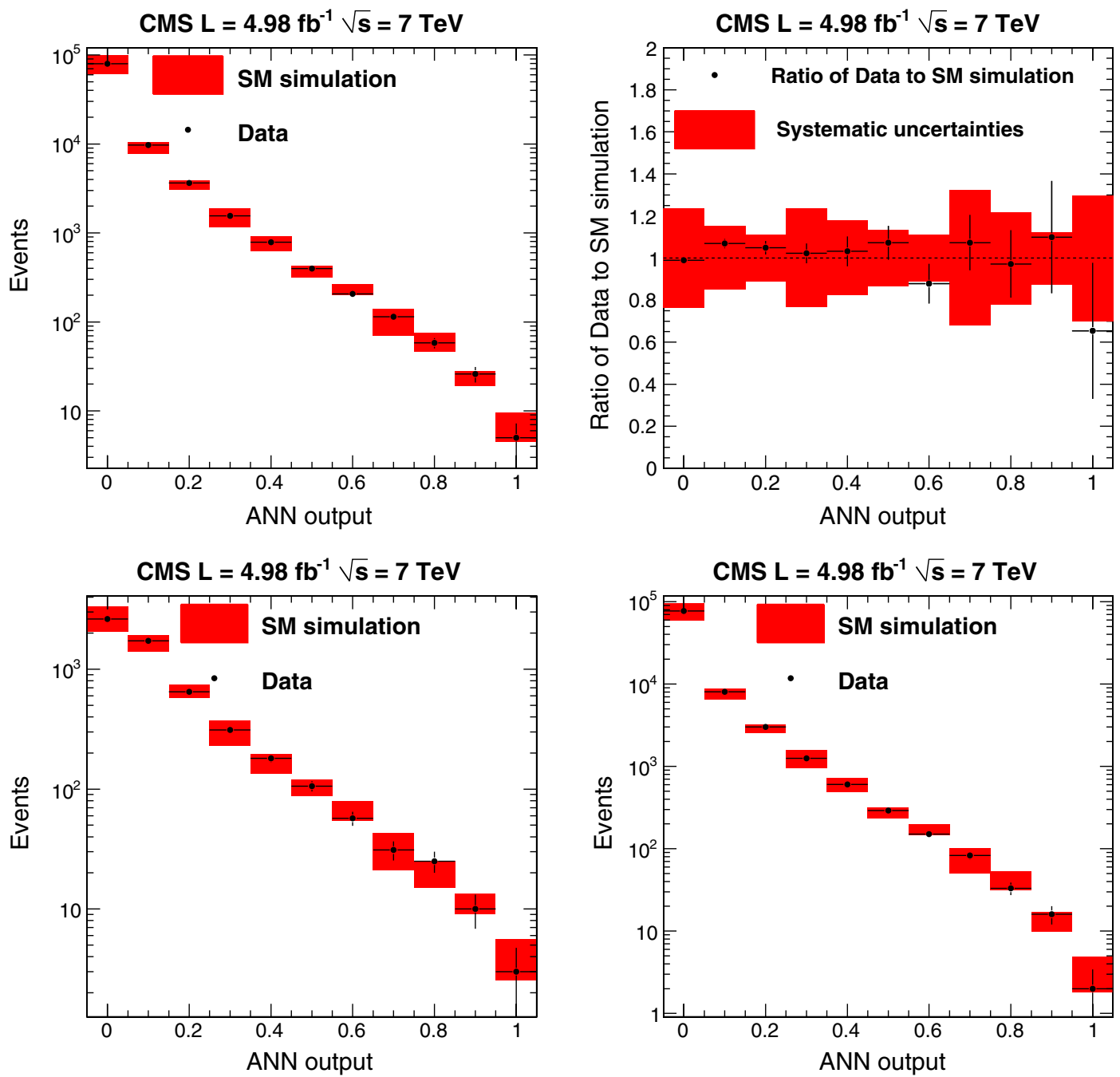

FIG. 2 (color online). Data (black points with error bars) vs simulated events (red bands) comparison of the ANN output distributions in the various control regions. Top: ANN output in the control region used to perform the extrapolation with systematic uncertainties included (left), and the ratio between data and simulated events (right) with both statistical (black error bars) and systematic (red bands) uncertainties shown. Bottom: main control region subdivided into the following two to perform cross-checks: $t \bar{t}$ enriched control region A (left), $Z+$ jet enriched control region B (right) with systematic uncertainties included.

TABLE III. Systematic uncertainties considered in the predicted background, along with their magnitude, and the impact they have on the final ANN output prediction when the signal selection requirement at 0.95 is applied.

\begin{tabular}{lcc}
\hline \hline Quantity & Syst. uncertainty & $\begin{array}{c}\text { Syst. error (\%) } \\
\text { on the SM prediction }\end{array}$ \\
\hline Missing transverse energy $\mathbb{E}_{\mathrm{T}}$ & $\pm 10 \%$ & 26 \\
Leading, subleading jet $p_{\mathrm{T}}$ & $\pm 3 \%$ & 10 \\
$\sum E_{\mathrm{T}}^{\text {lepton }}$ & $\pm 2 \%$ & 9 \\
$\sum_{\mathrm{T}} E_{\mathrm{T}}$ & $\pm 5 \%$ & 6 \\
Transverse mass $M_{\mathrm{T}}$ & $\pm 1 \%$ & 1 \\
Numberton mass $M_{\ell \ell}$ & $\pm 0.5 \%$ & $<1$ \\
$t \bar{t}$ cross section & $\pm 4 \%$ & $<1$ \\
QCD, $W+$ jets, $Z+$ jets cross sections & $\pm 50 \%, \pm 3 \%, \pm 3 \%$ & 30 \\
Total & & $<1$ \\
\hline \hline
\end{tabular}


TABLE IV. Systematic uncertainties on signal acceptance and efficiency.

\begin{tabular}{lc}
\hline \hline Source of systematic & Uncertainty \\
\hline Lepton triggers $\left(p_{\mathrm{T}}>20 \mathrm{GeV}\right)$ & $3 \%$ \\
Lepton isolation & $5 \%$ \\
Integrated luminosity & $2.2 \%$ \\
ANN selection & $17 \%$ \\
Total & $18 \%$ \\
\hline \hline
\end{tabular}

between data and simulation for the migration of events from the one-jet to the two-jet bin is estimated to be $0.5 \%$. Similarly, the systematic uncertainty on the ratio of the lepton to the total transverse energy is estimated to be $2 \%$, and the $M_{T}$ uncertainty is estimated to be $5 \%$. The dilepton mass scale uncertainty of $1 \%$ is taken from the CMS measurements of the $Z$ peak [32].

The relative fraction of $t \bar{t}$ and $Z+$ jets backgrounds is observed to vary as a function of the ANN output, as well as across the signal and control regions. In order to account for any remaining differences, the cross sections of all background components are left to vary within their uncertainties, taken from the recent CMS measurements for the $t \bar{t}[33]$ cross section, and using a conservative $50 \%$ uncertainty on the QCD cross section. The $Z+$ jet cross section uncertainty $(<3 \%)$ [32], and the $W+$ jet cross section uncertainty $<3 \%$ ) [32] produce a negligible systematic effect on the ANN output.

The systematic uncertainties associated with the signal acceptance and efficiency (ANN selection), along with their magnitude, are summarized in Table IV. The uncertainty on the lepton triggers and the lepton isolation are the same as the ones estimated in Ref. [34]. The relative ANN uncertainty for the signal is lower than the corresponding uncertainty for the background, due mainly to the different ANN shapes for these two populations (signal and background).

\section{PERFORMANCE OF THE ANN}

The ANN output after the training is shown in Fig. 3 for the signal (blue) and SM background (red) samples; the efficiency and purity of the selected samples are also shown as a function of the ANN output requirement.

When statistical and systematic uncertainties are taken into account, the ANN output requirement yielding the best expected exclusion limit in the SMS plane is ANN $>0.95$. The expected number of SM and signal events for the CMSSM benchmark point LM6 after imposing the ANN output requirement of $>0.95$ are shown in Table V. The remaining backgrounds are dominated by $t \bar{t}$ events in the dilepton final state, followed by $Z+$ jets production at a much smaller level.
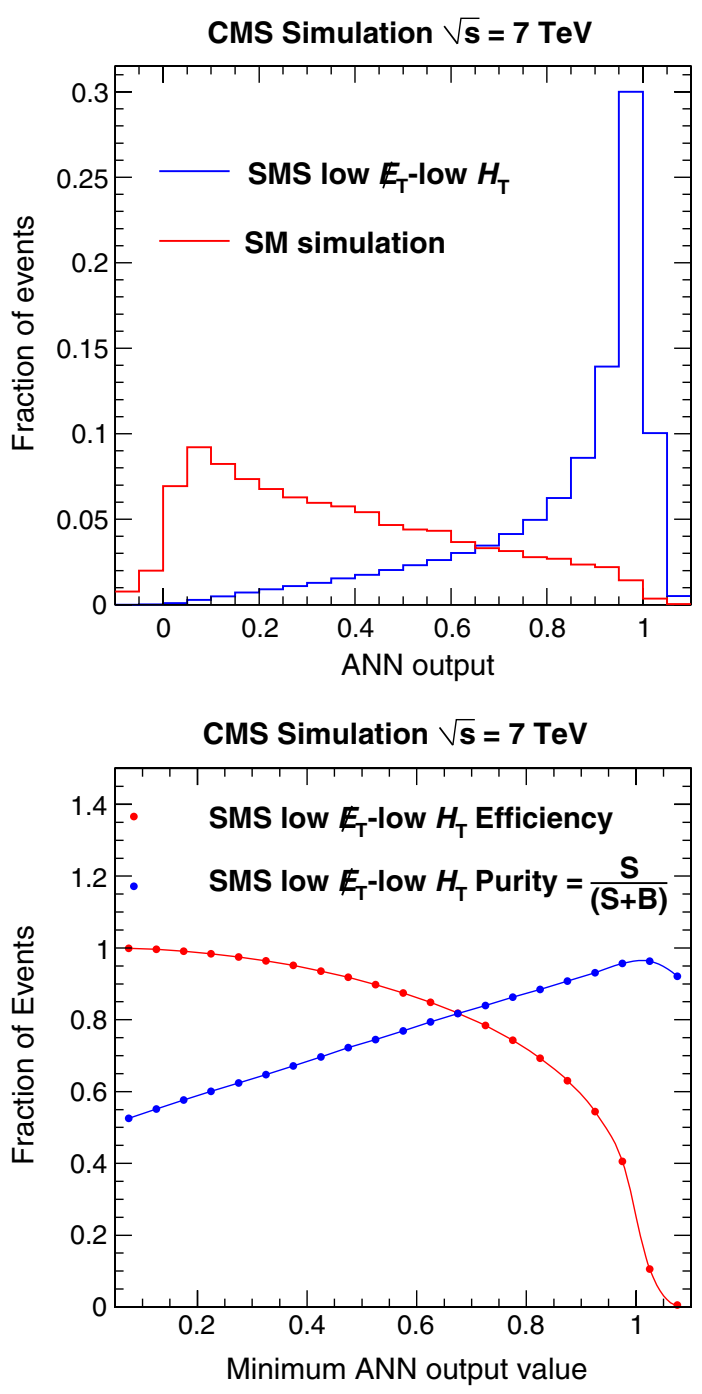

FIG. 3 (color online). Left: ANN output for the SM background (red bands) and SMS low $\not_{\mathrm{T}}$-low $H_{\mathrm{T}}$ events (blue line). Right: efficiency (red) and purity (blue) vs the minimum ANN output value for SMS low $\mathbb{E}_{\mathrm{T}}$-low $H_{\mathrm{T}}$ events in the signal region.

\section{RESULTS}

The seven input ANN variables are shown in Fig. 4 for simulated and data events, after the candidate event selection criteria are applied and for signal events. Data and

TABLE V. Expected number of events for signal and SM background and for the ANN output greater than $>0.95$. The NLO cross section is used for the CMSSM benchmark point LM6.

\begin{tabular}{lc}
\hline \hline Sample & Events with ANN $>0.95$ \\
\hline$t \bar{t}$ & $125 \pm 38$ \\
$Z+$ jets & $14 \pm 4$ \\
$W+$ jets, $W W, W Z, Z Z$, QCD & $<1$ \\
Total SM bkg. & $140 \pm 42$ \\
LM6 & $40 \pm 1$ \\
\hline \hline
\end{tabular}


simulation are consistent with each other, within the statistical and systematic uncertainties.

Figure 5 shows the comparison between the SM ANN prediction and the data in the signal region including statistical and systematic uncertainties.

In the signal-like region there are 171 events observed and $140_{-46}^{+73}$ (stat) \pm 42 (syst) expected. The statistical error on the expectation comes from the number of data events in the control region. The $95 \%$ confidence level (C.L.) upper limit on number of signal events is estimated to be 95 .
There is agreement between expectation and observation at a $68 \%$ C.L. Figure 6 shows the $\mathscr{E}_{\mathrm{T}}$ and $H_{\mathrm{T}}$ distributions for data and simulated events in the signal-like region. These figures illustrate that this analysis accepts signallike events with $\not_{\mathrm{T}}$ as low as $40 \mathrm{GeV}$ or $H_{\mathrm{T}}$ as low as $120 \mathrm{GeV}$-regions not explored yet by other CMS analyses.

Finally, the observed and expected number of events are translated into limits on SUSY parameter space. The 95\% C.L. upper limits are computed using a hybrid $\mathrm{CL}_{\mathrm{s}}$
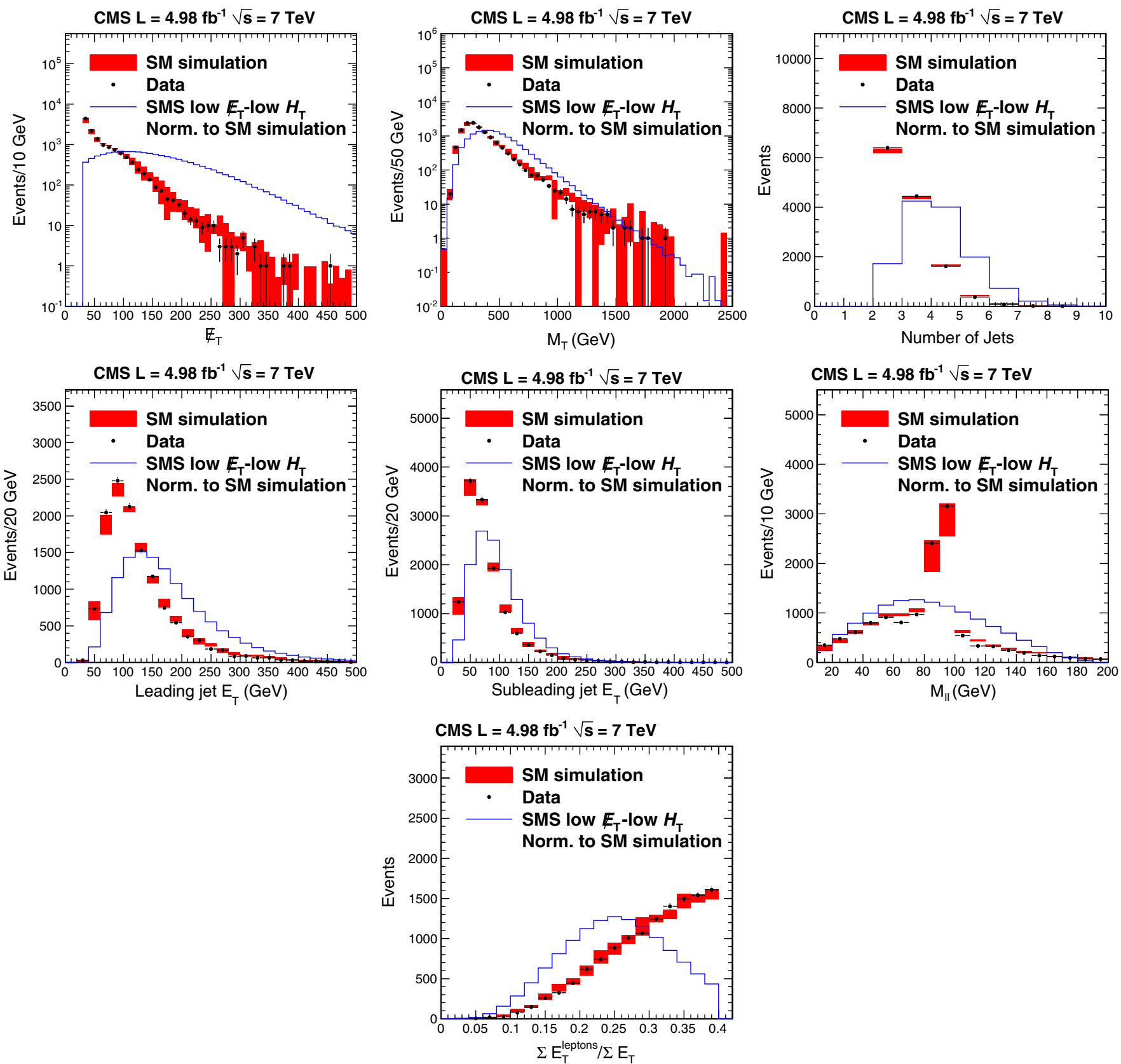

FIG. 4 (color online). Distributions in seven input ANN variables for simulated SM background events (red shaded band showing combined statistical and systematic uncertainties) and SMS low- $\mathbb{E}_{\mathrm{T}}$ low- $H_{\mathrm{T}}$ events (blue histogram), normalized to the same number of events. The data are also shown for comparison (black points with error bars). 


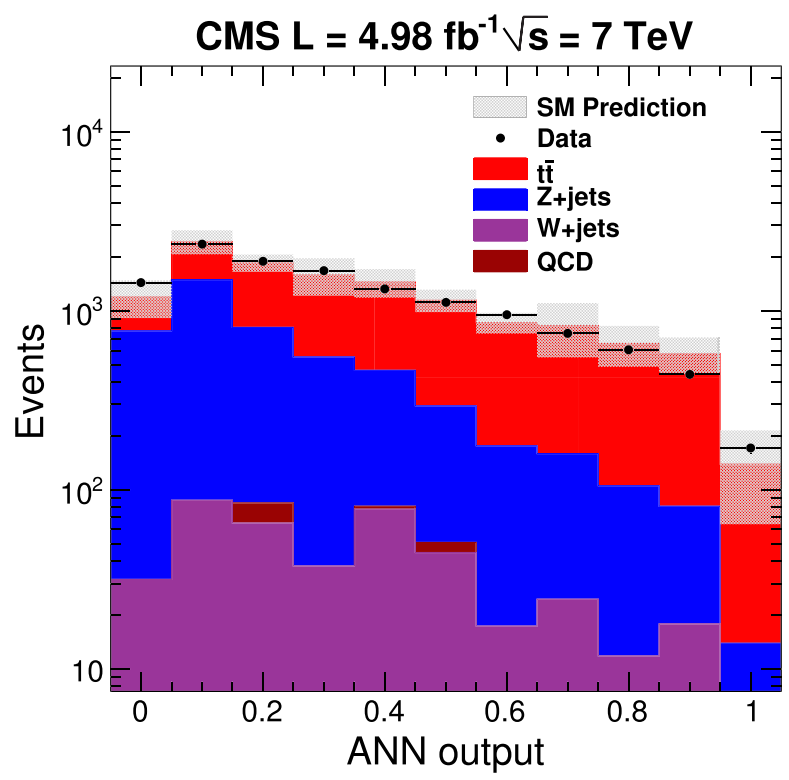

FIG. 5 (color online). ANN output for the data (black points) and the SM prediction based on control regions in data (gray band) in the signal region. The uncertainty on the SM prediction includes both statistical and systematic components. method with profile likelihood test statistics, and lognormal distributions for the background expectation [35,36]. The uncertainties in the NLO + NLL cross sections from the parton distribution functions [37-41], the choice of the factorization and renormalization scale, and $\alpha_{\mathrm{S}}$ are taken into account for each point and are evaluated according to the PDF4LHC recommendation [42]. A constant signal acceptance systematic uncertainty of $18 \%$ is assumed for each point. As described previously, the contamination of the signal in the control region is negligible and hence not taken into account in the limit setting.

The exclusion limits on SMS models are depicted in Fig. 7 , and in the $\left(m_{0}, m_{1 / 2}\right)$ CMSSM plane are shown in Fig. 8 [43].

As discussed earlier, for SUSY models that yield events with large $\mathbb{E}_{\mathrm{T}}$ (CMSSM with $\left.m_{0}<1000\right)$, the ANN's performance is comparable to the data analyses using large $\mathscr{E}_{\mathrm{T}}$ and $H_{\mathrm{T}}$, and in some cases worse, given that the ANN has been trained with models characterized by low $\mathbb{E}_{\mathrm{T}}$ and $H_{\mathrm{T}}$. For SUSY models that yield events with low $\not_{\mathrm{T}}$ and/or $H_{\mathrm{T}}$ (CMSSM with $m_{0}>1000$ and for SMS models close to the diagonal), the ANN's performance is better
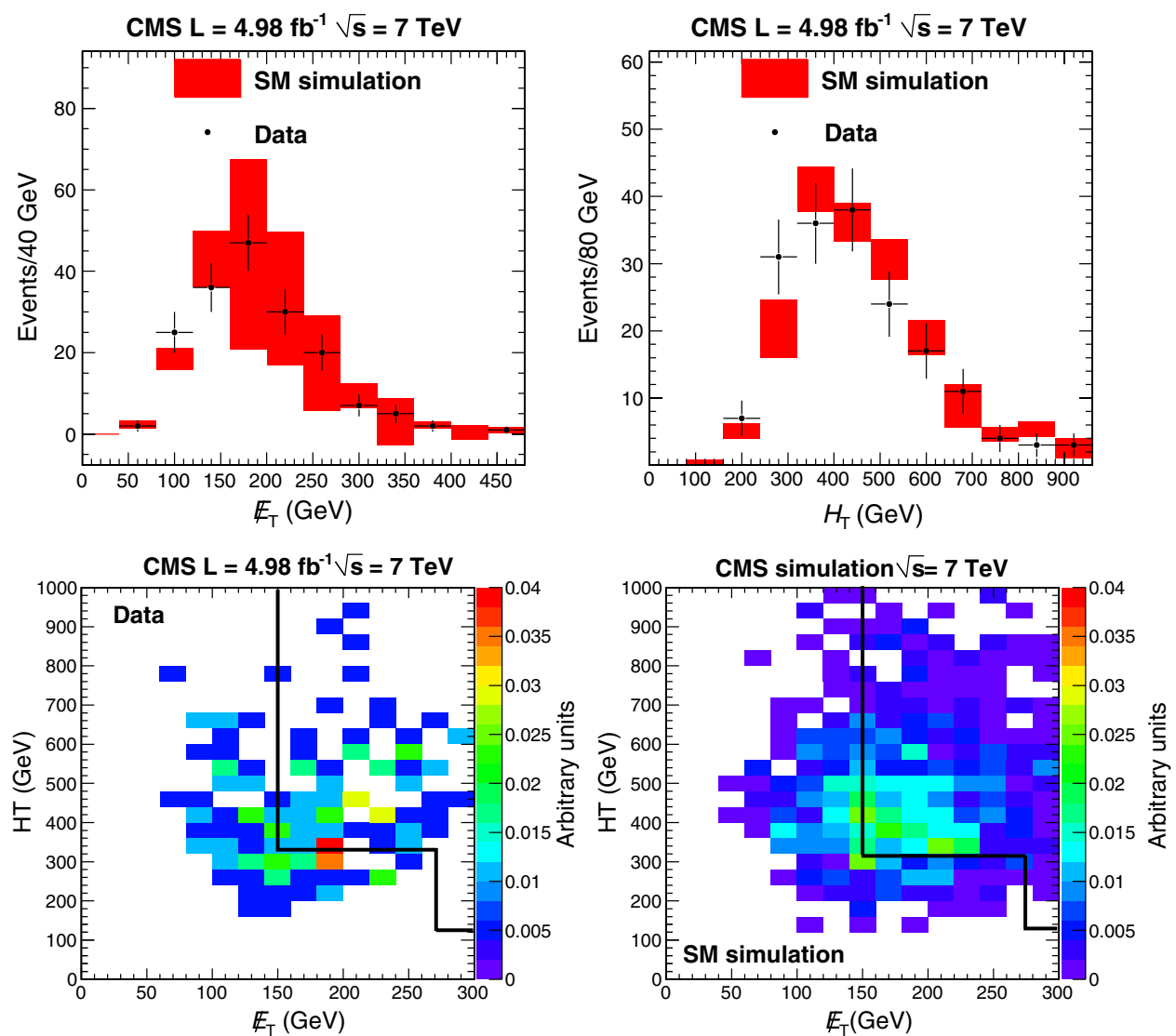

FIG. 6 (color online). Distribution in $\not_{\mathrm{T}}$ (top left) and $H_{\mathrm{T}}$ (top right) for signal-like events (ANN output $>0.95$ ) for data (black points with error bars) and simulation (red shaded band). The $\ddot{E}_{\mathrm{T}}$ vs $H_{\mathrm{T}}$ distributions for signal-like events (ANN $\left.>0.95\right)$ in data (bottom left) and simulation (bottom right); the regions to the right and up of the black line are the ones also explored by other CMS opposite-sign dilepton searches. 

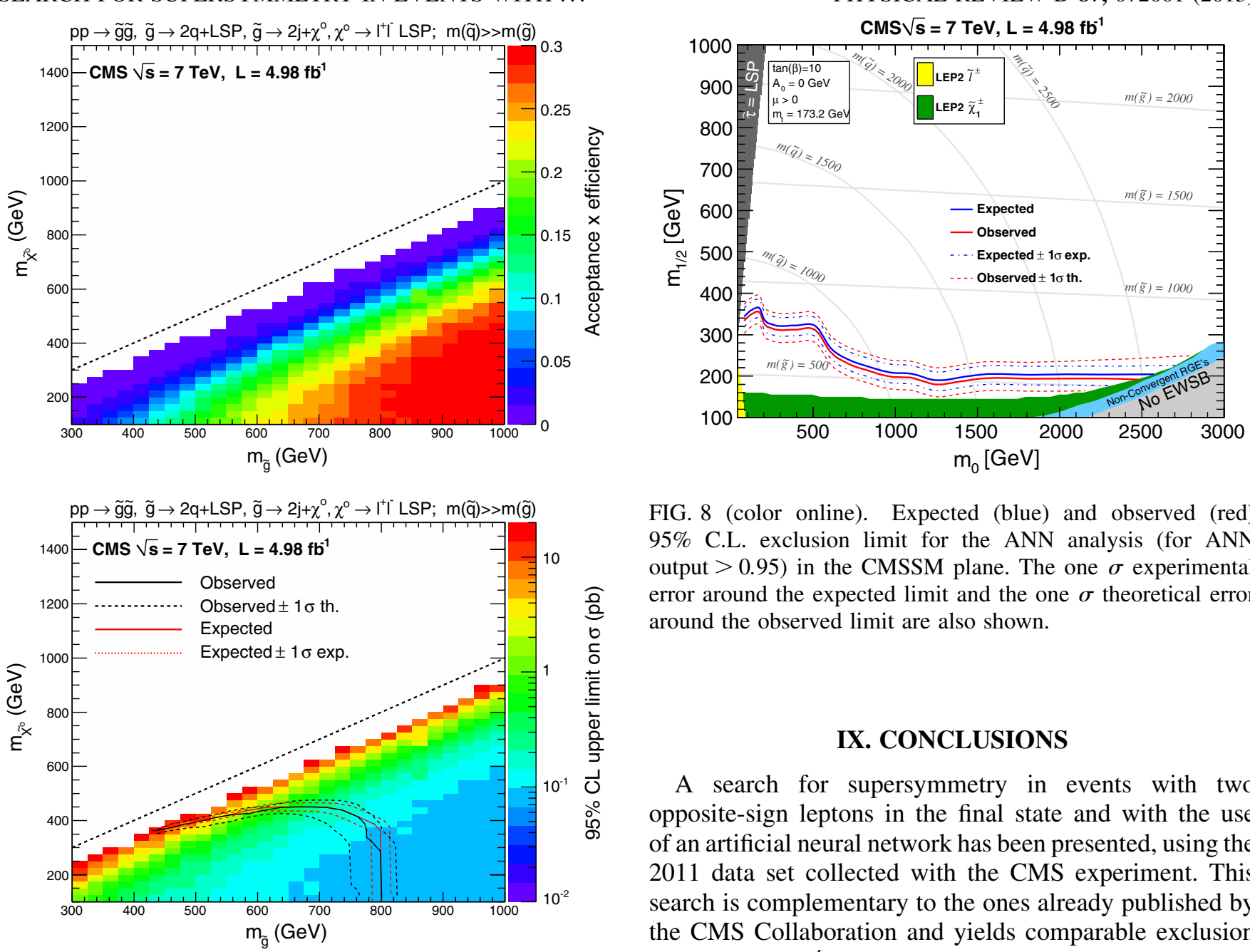

FIG. 8 (color online). Expected (blue) and observed (red) 95\% C.L. exclusion limit for the ANN analysis (for ANN output $>0.95$ ) in the CMSSM plane. The one $\sigma$ experimental error around the expected limit and the one $\sigma$ theoretical error around the observed limit are also shown.

\section{CONCLUSIONS}

A search for supersymmetry in events with two opposite-sign leptons in the final state and with the use of an artificial neural network has been presented, using the 2011 data set collected with the CMS experiment. This search is complementary to the ones already published by the CMS Collaboration and yields comparable exclusion limits for high- $\mathbb{E}_{\mathrm{T}}$, high- $H_{\mathrm{T}}$ SUSY models. In addition, the

FIG. 7 (color online). 95\% C.L. exclusion limits on the simplified model scenarios with the ANN analysis. The acceptance (fraction of events surviving event selection and candidate event selection) $\times$ efficiency (fraction of events surviving ANN selection) (top) and 95\% C.L. upper cross section limit (bottom) are shown for different gluino and neutralino masses. The region just below the diagonal is not considered due to inadequate initial state radiation modeling.

compared to the analyses using large $\mathbb{E}_{\mathrm{T}}$ and $H_{\mathrm{T}}$ selection criteria.

In the case of the CMSSM limits and for a specific choice of parameter values, squark masses below $\sim 700 \mathrm{GeV}$ are excluded at $95 \%$ C.L., and similarly gluino masses below $\sim 700 \mathrm{GeV}$ are excluded for the region $m_{0}<700 \mathrm{GeV}$. In the region $1000<m_{0}<3000 \mathrm{GeV}$, gluino masses below $\sim 300 \mathrm{GeV}$ are excluded, while the squark mass in the excluded models varies in the range from 1000 to $2500 \mathrm{GeV}$, depending on the value $m_{0}$. In the case of the SMS limits, for gluino masses below $\sim 800 \mathrm{GeV}$, LSP masses below $\sim 400 \mathrm{GeV}$ are excluded. For gluino masses above $\sim 800 \mathrm{GeV}$, no limits on the mass of LSP can be set. significantly relaxed criteria on $\mathscr{E}_{\mathrm{T}}$ and $H_{\mathrm{T}}$ with respect to the previously published analyses allows for the study of events not addressed by previous searches and provides an independent and complementary probe of this particularly challenging region of phase space. Agreement is observed between the expectation from the SM and the data, with no significant excess, which results in limits in the CMSSM $\left(m_{0}, m_{1 / 2}\right)$ and $\operatorname{SMS}\left(m_{\tilde{g}}, m_{\mathrm{LSP}}\right)$ planes.

\section{ACKNOWLEDGMENTS}

We congratulate our colleagues in the CERN accelerator departments for the excellent performance of the LHC and thank the technical and administrative staffs at CERN and at other CMS institutes for their contributions to the success of the CMS effort. In addition, we gratefully acknowledge the computing centers and personnel of the Worldwide LHC Computing Grid for delivering so effectively the computing infrastructure essential to our analyses. Finally, we acknowledge the enduring support for the construction and operation of the LHC and the CMS detector provided by the following funding agencies: the Austrian Federal Ministry of Science and Research; the Belgian Fonds de la Recherche Scientifique, and Fonds 
voor Wetenschappelijk Onderzoek; the Brazilian Funding Agencies (CNPq, CAPES, FAPERJ, and FAPESP); the Bulgarian Ministry of Education, Youth and Science; CERN; the Chinese Academy of Sciences, Ministry of Science and Technology, and National Natural Science Foundation of China; the Colombian Funding Agency (COLCIENCIAS); the Croatian Ministry of Science, Education and Sport; the Research Promotion Foundation, Cyprus; the Ministry of Education and Research, Recurrent financing Contract No. SF0690030s09 and European Regional Development Fund, Estonia; the Academy of Finland, Finnish Ministry of Education and Culture, and Helsinki Institute of Physics; the Institut National de Physique Nucléaire et de Physique des Particules/CNRS, and Commissariat à l'Énergie Atomique et aux Énergies Alternatives/CEA, France; the Bundesministerium für Bildung und Forschung, Deutsche Forschungsgemeinschaft, and Helmholtz-Gemeinschaft Deutscher Forschungszentren, Germany; the General Secretariat for Research and Technology, Greece; the National Scientific Research Foundation, and National Office for Research and Technology, Hungary; the Department of Atomic Energy and the Department of Science and Technology, India; the Institute for Studies in Theoretical Physics and Mathematics, Iran; the Science Foundation, Ireland; the Istituto Nazionale di Fisica Nucleare, Italy; the Korean Ministry of Education, Science and Technology and the World Class University program of NRF, Republic of Korea; the Lithuanian Academy of Sciences; the Mexican Funding Agencies (CINVESTAV, CONACYT, SEP, and UASLP-FAI); the Ministry of Science and Innovation, New Zealand; the Pakistan Atomic Energy Commission; the Ministry of Science and Higher
Education and the National Science Centre, Poland; the Fundação para a Ciência e a Tecnologia, Portugal; JINR (Armenia, Belarus, Georgia, Ukraine, Uzbekistan); the Ministry of Education and Science of the Russian Federation, the Federal Agency of Atomic Energy of the Russian Federation, Russian Academy of Sciences, and the Russian Foundation for Basic Research; the Ministry of Science and Technological Development of Serbia; the Secretaría de Estado de Investigación, Desarrollo e Innovación and Programa Consolider-Ingenio 2010, Spain; the Swiss Funding Agencies (ETH Board, ETH Zurich, PSI, SNF, UniZH, Canton Zurich, and SER); the National Science Council, Taipei; the Thailand Center of Excellence in Physics, the Institute for the Promotion of Teaching Science and Technology of Thailand and the National Science and Technology Development Agency of Thailand; the Scientific and Technical Research Council of Turkey, and Turkish Atomic Energy Authority; the Science and Technology Facilities Council, UK; the U.S. Department of Energy, and the U.S. National Science Foundation. Individuals have received support from the Marie-Curie program and the European Research Council (European Union); the Leventis Foundation; the A.P. Sloan Foundation; the Alexander von Humboldt Foundation; the Belgian Federal Science Policy Office; the Fonds pour la Formation à la Recherche dans l'Industrie et dans l'Agriculture (FRIA-Belgium); the Agentschap voor Innovatie door Wetenschap en Technologie (IWT-Belgium); the Ministry of Education, Youth and Sports (MEYS) of Czech Republic; the Council of Science and Industrial Research, India; the Compagnia di San Paolo (Torino); and the HOMING PLUS program of Foundation for Polish Science, co-financed from European Union, Regional Development Fund.
[1] Y. A. Golfand and E. P. Likhtman, JETP Lett. 13, 323 (1971) [http://www.jetpletters.ac.ru/ps/1584/article_24309 .shtml.

[2] J. Wess and B. Zumino, Nucl. Phys. B70, 39 (1974).

[3] H. P. Nilles, Phys. Rep. 110, 1 (1984).

[4] H. E. Haber and G. L. Kane, Phys. Rep. 117, 75 (1985).

[5] R. Barbieri, S. Ferrara, and C. A. Savoy, Phys. Lett. 119B, 343 (1982).

[6] S. Dawson, E. Eichten, and C. Quigg, Phys. Rev. D 31, 1581 (1985).

[7] E. Witten, Nucl. Phys. B188, 513 (1981).

[8] S. Dimopoulos and H. Georgi, Nucl. Phys. B193, 150 (1981).

[9] G. Jungman and M. Kamionkowski, Phys. Rep. 267, 195 (1996).

[10] G. R. Farrar and P. Fayet, Phys. Lett. B76, 575 (1978).

[11] CMS Collaboration, Phys. Lett. B 718, 815 (2013).

[12] CMS Collaboration, Phys. Lett. B 716, 260 (2012).
[13] G. L. Kane, C. Kolda, L. Roszkowski, and J.D. Wells, Phys. Rev. D 49, 6173 (1994).

[14] A. H. Chamseddine, R. L. Arnowitt, and P. Nath, Phys. Rev. Lett. 49, 970 (1982).

[15] N. Arkani-Hamed, P. Schuster, N. Toro, J. Thaler, L.-T. Wang, B. Knuteson, and S. Mrenna, arXiv:hep-ph/ 0703088.

[16] D. Alves et al., J. Phys. G 39, 105005 (2012).

[17] CMS Collaboration, JINST 3, S08003 (2008).

[18] CMS Collaboration, CMS Report No. CERN-LHCC 2000-038; No. CMS-TDR-006-1, 2000, http://cds.cern.ch/ record/706847? $1 n=e n$.

[19] CMS Collaboration, Eur. Phys. J. C 46, 605 (2006).

[20] CMS Collaboration, JINST 7, P10002 (2012).

[21] CMS Collaboration, CMS Report No. CMS-PAS-EGM10-004, 2010, http://cds.cern.ch/record/1299116?ln=en.

[22] M. Cacciari, G. P. Salam, and G. Soyez, J. High Energy Phys. 04 (2008) 063. 
[23] CMS Collaboration, CMS Report No. CMS-PAS-PFT-09001, 2009, http://cds.cern.ch/record/1194487?!n=en.

[24] CMS Collaboration, JINST 6, P11002 (2011).

[25] T. Sjöstrand, S. Mrenna, and P. Z. Skands, J. High Energy Phys. 05 (2006) 026.

[26] R. Field, arXiv: 1010.3558.

[27] J. Alwall, P. Demin, S. de Visscher, R. Frederix, M. Herquet, F. Maltoni, T. Plehn, D.L. Rainwater, and T. Stelzer, J. High Energy Phys. 09 (2007) 028.

[28] S. Agostinelli et al., Nucl. Instrum. Methods Phys. Res., Sect. A 506, 250 (2003).

[29] CMS Collaboration, CMS Report No. CMS-PAS-TRK10-005, 2010, http://cds.cern.ch/record/1279383? In=en.

[30] CMS Collaboration, CMS Report No. CMS-PAS-JME-09008, 2010, http://cds.cern.ch/record/1259924?ln=en.

[31] CMS Collaboration, JINST 6, P09001 (2011).

[32] CMS Collaboration, J. High Energy Phys. 10 (2011) 132.

[33] CMS Collaboration, J. High Energy Phys. 07 (2011) 049.
[34] CMS Collaboration, Phys. Rev. Lett. 109, 071803 (2012).

[35] A. L. Read, J. Phys. G 28, 2693 (2002).

[36] T. Junk, Nucl. Instrum. Methods Phys. Res., Sect. A 434, 435 (1999).

[37] M. Krämer, A. Kulesza, R. van der Leeuw, M. Mangano, S. Padhi, T. Plehn, and X. Portell, arXiv:1206.2892.

[38] W. Beenakker, R. Höpker, M. Spira, and P. M. Zerwas, Nucl. Phys. B492, 51 (1997).

[39] A. Kulesza and L. Motyka, Phys. Rev. Lett. 102, 111802 (2009).

[40] A. Kulesza and L. Motyka, Phys. Rev. D 80, 095004 (2009).

[41] W. Beenakker, S. Brensing, M. Krämer, A. Kulesza, E. Laenen, and I. Niessen, J. High Energy Phys. 12 (2009) 041.

[42] S. Alekhin et al., arXiv:1101.0536.

[43] K. Matchev and R. Remington, arXiv:1202.6580.

S. Chatrchyan, ${ }^{1}$ V. Khachatryan, ${ }^{1}$ A. M. Sirunyan, ${ }^{1}$ A. Tumasyan, ${ }^{1}$ W. Adam,${ }^{2}$ E. Aguilo, ${ }^{2}$ T. Bergauer, ${ }^{2}$

M. Dragicevic, ${ }^{2}$ J. Erö, ${ }^{2}$ C. Fabjan, ${ }^{2, b}$ M. Friedl, ${ }^{2}$ R. Frühwirth, ${ }^{2, b}$ V. M. Ghete, ${ }^{2}$ N. Hörmann, ${ }^{2}$ J. Hrubec, ${ }^{2}$ M. Jeitler, ${ }^{2, b}$ W. Kiesenhofer, ${ }^{2}$ V. Knünz, ${ }^{2}$ M. Krammer, ${ }^{2, b}$ I. Krätschmer, ${ }^{2}$ D. Liko, ${ }^{2}$ I. Mikulec, ${ }^{2}$ M. Pernicka, ${ }^{2, a}$ D. Rabady, ${ }^{2, \mathrm{c}}$ B. Rahbaran, ${ }^{2}$ C. Rohringer, ${ }^{2}$ H. Rohringer, ${ }^{2}$ R. Schöfbeck, ${ }^{2}$ J. Strauss, ${ }^{2}$ A. Taurok, ${ }^{2}$ W. Waltenberger, ${ }^{2}$ C.-E. Wulz, ${ }^{2, b}$ V. Mossolov, ${ }^{3}$ N. Shumeiko, ${ }^{3}$ J. Suarez Gonzalez, ${ }^{3}$ M. Bansal,${ }^{4}$ S. Bansal, ${ }^{4}$ T. Cornelis, ${ }^{4}$ E. A. De Wolf, ${ }^{4}$ X. Janssen, ${ }^{4}$ S. Luyckx,${ }^{4}$ L. Mucibello, ${ }^{4}$ S. Ochesanu, ${ }^{4}$ B. Roland, ${ }^{4}$ R. Rougny, ${ }^{4}$ M. Selvaggi, ${ }^{4}$ H. Van Haevermaet, ${ }^{4}$ P. Van Mechelen, ${ }^{4}$ N. Van Remortel, ${ }^{4}$ A. Van Spilbeeck, ${ }^{4}$ F. Blekman, ${ }^{5}$ S. Blyweert,${ }^{5}$ J. D'Hondt, ${ }^{5}$ R. Gonzalez Suarez, ${ }^{5}$ A. Kalogeropoulos, ${ }^{5}$ M. Maes, ${ }^{5}$ A. Olbrechts, ${ }^{5}$ W. Van Doninck, ${ }^{5}$ P. Van Mulders, ${ }^{5}$ G. P. Van Onsem, ${ }^{5}$ I. Villella, ${ }^{5}$ B. Clerbaux, ${ }^{6}$ G. De Lentdecker, ${ }^{6}$ V. Dero, ${ }^{6}$ A. P. R. Gay, ${ }^{6}$ T. Hreus, ${ }^{6}$ A. Léonard, ${ }^{6}$ P. E. Marage, ${ }^{6}$ A. Mohammadi, ${ }^{6}$ T. Reis,${ }^{6}$ L. Thomas,${ }^{6}$ C. Vander Velde, ${ }^{6}$ P. Vanlaer, ${ }^{6}$ J. Wang, ${ }^{6}$ V. Adler, ${ }^{7}$ K. Beernaert, ${ }^{7}$ A. Cimmino, ${ }^{7}$ S. Costantini, ${ }^{7}$ G. Garcia, ${ }^{7}$ M. Grunewald, ${ }^{7}$ B. Klein, ${ }^{7}$ J. Lellouch, ${ }^{7}$ A. Marinov, ${ }^{7}$ J. Mccartin, ${ }^{7}$ A. A. Ocampo Rios, ${ }^{7}$ D. Ryckbosch, ${ }^{7}$ N. Strobbe, ${ }^{7}$ F. Thyssen, ${ }^{7}$ M. Tytgat, ${ }^{7}$ S. Walsh, ${ }^{7}$ E. Yazgan, ${ }^{7}$ N. Zaganidis, ${ }^{7}$ S. Basegmez ${ }^{8}$ G. Bruno, ${ }^{8}$ R. Castello, ${ }^{8}$ L. Ceard,${ }^{8}$ C. Delaere, ${ }^{8}$ T. du Pree,${ }^{8}$ D. Favart, ${ }^{8}$ L. Forthomme, ${ }^{8}$ A. Giammanco, ${ }^{8, \mathrm{~d}}$ J. Hollar, ${ }^{8}$ V. Lemaitre, ${ }^{8}$ J. Liao, ${ }^{8}$ O. Militaru, ${ }^{8}$ C. Nuttens, ${ }^{8}$ D. Pagano, ${ }^{8}$ A. Pin, ${ }^{8}$ K. Piotrzkowski, ${ }^{8}$ J. M. Vizan Garcia, ${ }^{8}$ N. Beliy, ${ }^{9}$ T. Caebergs, ${ }^{9}$ E. Daubie, ${ }^{9}$ G. H. Hammad, ${ }^{9}$ G. A. Alves,${ }^{10}$ M. Correa Martins Junior, ${ }^{10}$ T. Martins,${ }^{10}$ M.E. Pol, ${ }^{10}$ M. H. G. Souza, ${ }^{10}$ W. L. Aldá Júnior,${ }^{11}$ W. Carvalho,,${ }^{11}$ A. Custódio, ${ }^{11}$ E. M. Da Costa, ${ }^{11}$ D. De Jesus Damiao, ${ }^{11}$ C. De Oliveira Martins, ${ }^{11}$ S. Fonseca De Souza,${ }^{11}$ H. Malbouisson, ${ }^{11}$ M. Malek, ${ }^{11}$ D. Matos Figueiredo, ${ }^{11}$ L. Mundim,,${ }^{11}$ H. Nogima, ${ }^{11}$ W. L. Prado Da Silva, ${ }^{11}$ A. Santoro, ${ }^{11}$ L. Soares Jorge, ${ }^{11}$ A. Sznajder, ${ }^{11}$ A. Vilela Pereira, ${ }^{11}$ T. S. Anjos, ${ }^{12 b}$ C. A. Bernardes, ${ }^{12 b}$ F. A. Dias, ${ }^{12 a, e}$ T. R. Fernandez Perez Tomei, ${ }^{12 a}$ E. M. Gregores, ${ }^{12 b}$ C. Lagana, ${ }^{12 a}$ F. Marinho, ${ }^{12 a}$ P. G. Mercadante, ${ }^{12 b}$

S. F. Novaes, ${ }^{12 a}$ Sandra S. Padula, ${ }^{12 a}$ V. Genchev, ${ }^{13, c}$ P. Iaydjiev, ${ }^{13, c}$ S. Piperov, ${ }^{13}$ M. Rodozov ${ }^{13}$ S. Stoykova, ${ }^{13}$

G. Sultanov, ${ }^{13}$ V. Tcholakov, ${ }^{13}$ R. Trayanov, ${ }^{13}$ M. Vutova, ${ }^{13}$ A. Dimitrov, ${ }^{14}$ R. Hadjiiska, ${ }^{14}$ V. Kozhuharov, ${ }^{14}$ L. Litov, ${ }^{14}$ B. Pavlov, ${ }^{14}$ P. Petkov, ${ }^{14}$ J. G. Bian, ${ }^{15}$ G. M. Chen, ${ }^{15}$ H. S. Chen, ${ }^{15}$ C. H. Jiang, ${ }^{15}$ D. Liang, ${ }^{15}$ S. Liang, ${ }^{15}$ X. Meng, ${ }^{15}$ J. Tao, ${ }^{15}$ J. Wang, ${ }^{15}$ X. Wang, ${ }^{15}$ Z. Wang, ${ }^{15}$ H. Xiao, ${ }^{15}$ M. Xu, ${ }^{15}$ J. Zang, ${ }^{15}$ Z. Zhang, ${ }^{15}$ C. Asawatangtrakuldee, ${ }^{16}$ Y. Ban, ${ }^{16}$ Y. Guo,${ }^{16}$ W. Li,${ }^{16}$ S. Liu, ${ }^{16}$ Y. Mao, ${ }^{16}$ S. J. Qian, ${ }^{16}$ H. Teng, ${ }^{16}$ D. Wang, ${ }^{16}$ L. Zhang, ${ }^{16}$ W. Zou, ${ }^{16}$ C. Avila, ${ }^{17}$ J. P. Gomez, ${ }^{17}$ B. Gomez Moreno, ${ }^{17}$ A. F. Osorio Oliveros, ${ }^{17}$ J. C. Sanabria, ${ }^{17}$ N. Godinovic, ${ }^{18}$ D. Lelas, ${ }^{18}$ R. Plestina, ${ }^{18, f}$ D. Polic,${ }^{18}$ I. Puljak, ${ }^{18, c}$ Z. Antunovic, ${ }^{19}$ M. Kovac, ${ }^{19}$ V. Brigljevic,${ }^{20}$ S. Duric,${ }^{20}$ K. Kadija,${ }^{20}$ J. Luetic,${ }^{20}$ D. Mekterovic,${ }^{20}$ S. Morovic, ${ }^{20}$ A. Attikis, ${ }^{21}$ M. Galanti, ${ }^{21}$ G. Mavromanolakis, ${ }^{21}$ J. Mousa, ${ }^{21}$ C. Nicolaou, ${ }^{21}$ F. Ptochos,${ }^{21}$ P. A. Razis, ${ }^{21}$ M. Finger, ${ }^{22}$ M. Finger, Jr. ${ }^{22}$ Y. Assran, ${ }^{23, g}$ S. Elgammal, ${ }^{23, h}$ A. Ellithi Kamel, ${ }^{23, \mathrm{i}}$ M. A. Mahmoud ${ }^{23, \mathrm{j}}$ A. Mahrous, ${ }^{23, \mathrm{k}}$ A. Radi,${ }^{23,1, \mathrm{~m}}$ M. Kadastik, ${ }^{24}$ M. Müntel, ${ }^{24}$ M. Murumaa ${ }^{24}$ M. Raidal,${ }^{24}$ L. Rebane,${ }^{24}$ A. Tiko, ${ }^{24}$ P. Eerola,${ }^{25}$ G. Fedi, ${ }^{25}$ M. Voutilainen,${ }^{25}$ J. Härkönen,${ }^{26}$ A. Heikkinen, ${ }^{26}$ V. Karimäki, ${ }^{26}$ R. Kinnunen, ${ }^{26}$ M. J. Kortelainen, ${ }^{26}$ T. Lampén, ${ }^{26}$ K. Lassila-Perini, ${ }^{26}$ S. Lehti, ${ }^{26}$ T. Lindén, ${ }^{26}$ P. Luukka, ${ }^{26}$ T. Mäenpää, ${ }^{26}$ T. Peltola,${ }^{26}$ E. Tuominen, ${ }^{26} \mathrm{~J}$. Tuominiemi, ${ }^{26}$ E. Tuovinen,${ }^{26}$ D. Ungaro, ${ }^{26}$ L. Wendland, ${ }^{26}$ K. Banzuzi, ${ }^{27}$ A. Karjalainen, ${ }^{27}$ A. Korpela, ${ }^{27}$ T. Tuuva, ${ }^{27}$ M. Besancon, ${ }^{28}$ S. Choudhury, ${ }^{28}$ 
M. Dejardin, ${ }^{28}$ D. Denegri, ${ }^{28}$ B. Fabbro, ${ }^{28}$ J. L. Faure, ${ }^{28}$ F. Ferri, ${ }^{28}$ S. Ganjour, ${ }^{28}$ A. Givernaud,${ }^{28}$ P. Gras, ${ }^{28}$ G. Hamel de Monchenault, ${ }^{28}$ P. Jarry, ${ }^{28}$ E. Locci, ${ }^{28}$ J. Malcles, ${ }^{28}$ L. Millischer, ${ }^{28}$ A. Nayak, ${ }^{28}$ J. Rander ${ }^{28}$ A. Rosowsky, ${ }^{28}$ M. Titov, ${ }^{28}$ S. Baffioni, ${ }^{29}$ F. Beaudette,${ }^{29}$ L. Benhabib,${ }^{29}$ L. Bianchini, ${ }^{29}$ M. Bluj, ${ }^{29, n}$ P. Busson, ${ }^{29}$ C. Charlot, ${ }^{29}$ N. Daci ${ }^{29}$ T. Dahms,${ }^{29}$ M. Dalchenko, ${ }^{29}$ L. Dobrzynski, ${ }^{29}$ A. Florent, ${ }^{29}$ R. Granier de Cassagnac, ${ }^{29}$ M. Haguenauer, ${ }^{29}$ P. Miné, ${ }^{29}$ C. Mironov, ${ }^{29}$ I. N. Naranjo, ${ }^{29}$ M. Nguyen, ${ }^{29}$ C. Ochando,${ }^{29}$ P. Paganini, ${ }^{29}$ D. Sabes ${ }^{29}$ R. Salerno, ${ }^{29}$ Y. Sirois, ${ }^{29}$ C. Veelken, ${ }^{29}$ A. Zabi, ${ }^{29}$ J.-L. Agram, ${ }^{30,0}$ J. Andrea, ${ }^{30}$ D. Bloch,${ }^{30}$ D. Bodin, ${ }^{30}$ J.-M. Brom, ${ }^{30}$ M. Cardaci, ${ }^{30}$ E. C. Chabert, ${ }^{30}$ C. Collard ${ }^{30}$ E. Conte,${ }^{30, o}$ F. Drouhin,${ }^{30, o}$ J.-C. Fontaine, ${ }^{30, o}$ D. Gelé, ${ }^{30}$ U. Goerlach, ${ }^{30}$ P. Juillot, ${ }^{30}$ A.-C. Le Bihan, ${ }^{30}$ P. Van Hove,${ }^{30}$ F. Fassi, ${ }^{31}$ D. Mercier,${ }^{31}$ S. Beauceron, ${ }^{32}$ N. Beaupere, ${ }^{32}$ O. Bondu, ${ }^{32}$ G. Boudoul, ${ }^{32}$ S. Brochet, ${ }^{32}$ J. Chasserat,${ }^{32}$ R. Chierici, ${ }^{32, c}$ D. Contardo, ${ }^{32}$ P. Depasse, ${ }^{32}$

H. El Mamouni, ${ }^{32}$ J. Fay, ${ }^{32}$ S. Gascon, ${ }^{32}$ M. Gouzevitch, ${ }^{32}$ B. Ille, ${ }^{32}$ T. Kurca, ${ }^{32}$ M. Lethuillier, ${ }^{32}$ L. Mirabito, ${ }^{32}$ S. Perries, ${ }^{32}$ L. Sgandurra, ${ }^{32}$ V. Sordini, ${ }^{32}$ Y. Tschudi, ${ }^{32}$ P. Verdier, ${ }^{32}$ S. Viret, ${ }^{32}$ Z. Tsamalaidze, ${ }^{33, p}$ C. Autermann, ${ }^{34}$ S. Beranek, ${ }^{34}$ B. Calpas, ${ }^{34}$ M. Edelhoff, ${ }^{34}$ L. Feld,${ }^{34}$ N. Heracleous, ${ }^{34}$ O. Hindrichs,${ }^{34}$ R. Jussen, ${ }^{34}$ K. Klein, ${ }^{34}$ J. Merz, ${ }^{34}$ A. Ostapchuk,${ }^{34}$ A. Perieanu,${ }^{34}$ F. Raupach,${ }^{34}$ J. Sammet, ${ }^{34}$ S. Schael,,${ }^{34}$ D. Sprenger, ${ }^{34}$ H. Weber,${ }^{34}$ B. Wittmer, ${ }^{34}$ V. Zhukov ${ }^{34, q}$ M. Ata,${ }^{35}$ J. Caudron, ${ }^{35}$ E. Dietz-Laursonn, ${ }^{35}$ D. Duchardt,${ }^{35}$ M. Erdmann,${ }^{35}$

R. Fischer, ${ }^{35}$ A. Güth, ${ }^{35}$ T. Hebbeker, ${ }^{35}$ C. Heidemann, ${ }^{35}$ K. Hoepfner, ${ }^{35}$ D. Klingebiel, ${ }^{35}$ P. Kreuzer, ${ }^{35}$

M. Merschmeyer, ${ }^{35}$ A. Meyer, ${ }^{35}$ M. Olschewski, ${ }^{35}$ P. Papacz, ${ }^{35}$ H. Pieta,${ }^{35}$ H. Reithler, ${ }^{35}$ S. A. Schmitz, ${ }^{35}$ L. Sonnenschein, ${ }^{35}$ J. Steggemann, ${ }^{35}$ D. Teyssier, ${ }^{35}$ S. Thüer, ${ }^{35}$ M. Weber,${ }^{35}$ M. Bontenackels, ${ }^{36}$ V. Cherepanov, ${ }^{36}$ Y. Erdogan, ${ }^{36}$ G. Flügge, ${ }^{36}$ H. Geenen, ${ }^{36}$ M. Geisler, ${ }^{36}$ W. Haj Ahmad,${ }^{36}$ F. Hoehle,${ }^{36}$ B. Kargoll,${ }^{36}$ T. Kress, ${ }^{36}$

Y. Kuessel, ${ }^{36}$ J. Lingemann, ${ }^{36, c}$ A. Nowack,${ }^{36}$ L. Perchalla, ${ }^{36}$ O. Pooth, ${ }^{36}$ P. Sauerland, ${ }^{36}$ A. Stahl, ${ }^{36}$

M. Aldaya Martin, ${ }^{37}$ J. Behr,${ }^{37}$ W. Behrenhoff,,${ }^{37}$ U. Behrens,${ }^{37}$ M. Bergholz,${ }^{37, r}$ A. Bethani, ${ }^{37} \mathrm{~K}$. Borras,${ }^{37}$

A. Burgmeier, ${ }^{37}$ A. Cakir,${ }^{37}$ L. Calligaris,${ }^{37}$ A. Campbell, ${ }^{37}$ E. Castro, ${ }^{37}$ F. Costanza, ${ }^{37}$ D. Dammann, ${ }^{37}$ C. Diez Pardos, ${ }^{37}$ G. Eckerlin, ${ }^{37}$ D. Eckstein, ${ }^{37}$ G. Flucke, ${ }^{37}$ A. Geiser, ${ }^{37}$ I. Glushkov, ${ }^{37}$ P. Gunnellini, ${ }^{37}$ S. Habib,${ }^{37}$ J. Hauk, ${ }^{37}$ G. Hellwig, ${ }^{37}$ H. Jung, ${ }^{37}$ M. Kasemann, ${ }^{37}$ P. Katsas, ${ }^{37}$ C. Kleinwort, ${ }^{37}$ H. Kluge, ${ }^{37}$ A. Knutsson, ${ }^{37}$ M. Krämer, ${ }^{37}$ D. Krücker, ${ }^{37}$ E. Kuznetsova, ${ }^{37}$ W. Lange, ${ }^{37}$ J. Leonard ${ }^{37}$ W. Lohmann, ${ }^{37, r}$ B. Lutz, ${ }^{37}$ R. Mankel, ${ }^{37}$ I. Marfin, ${ }^{37}$ M. Marienfeld, ${ }^{37}$ I.-A. Melzer-Pellmann, ${ }^{37}$ A. B. Meyer, ${ }^{37}$ J. Mnich, ${ }^{37}$ A. Mussgiller, ${ }^{37}$ S. Naumann-Emme, ${ }^{37}$ O. Novgorodova, ${ }^{37}$ J. Olzem, ${ }^{37}$ H. Perrey,${ }^{37}$ A. Petrukhin, ${ }^{37}$ D. Pitzl, ${ }^{37}$ A. Raspereza, ${ }^{37}$ P. M. Ribeiro Cipriano, ${ }^{37}$ C. Riedl, ${ }^{37}$ E. Ron, ${ }^{37}$ M. Rosin,,${ }^{37}$ J. Salfeld-Nebgen,${ }^{37}$ R. Schmidt, ${ }^{37, r}$

T. Schoerner-Sadenius, ${ }^{37}$ N. Sen, ${ }^{37}$ A. Spiridonov, ${ }^{37}$ M. Stein, ${ }^{37}$ R. Walsh, ${ }^{37}$ C. Wissing,${ }^{37}$ V. Blobel,${ }^{38}$ H. Enderle, ${ }^{38}$ J. Erfle, ${ }^{38}$ U. Gebbert, ${ }^{38}$ M. Görner, ${ }^{38}$ M. Gosselink, ${ }^{38}$ J. Haller, ${ }^{38}$ T. Hermanns, ${ }^{38}$ R. S. Höing, ${ }^{38}$ K. Kaschube, ${ }^{38}$ G. Kaussen, ${ }^{38}$ H. Kirschenmann, ${ }^{38}$ R. Klanner, ${ }^{38}$ J. Lange, ${ }^{38}$ F. Nowak, ${ }^{38}$ T. Peiffer,${ }^{38}$ N. Pietsch, ${ }^{38}$ D. Rathjens, ${ }^{38}$ C. Sander,${ }^{38}$ H. Schettler, ${ }^{38}$ P. Schleper, ${ }^{38}$ E. Schlieckau, ${ }^{38}$ A. Schmidt, ${ }^{38}$ M. Schröder, ${ }^{38}$ T. Schum, ${ }^{38}$ M. Seidel, ${ }^{38}$ J. Sibille, ${ }^{38, s}$ V. Sola ${ }^{38}$ H. Stadie, ${ }^{38}$ G. Steinbrück, ${ }^{38}$ J. Thomsen, ${ }^{38}$ L. Vanelderen, ${ }^{38}$ C. Barth,${ }^{39}$ J. Berger, ${ }^{39}$ C. Böser, ${ }^{39}$ T. Chwalek, ${ }^{39}$ W. De Boer, ${ }^{39}$ A. Descroix,${ }^{39}$ A. Dierlamm, ${ }^{39}$ M. Feindt, ${ }^{39}$ M. Guthoff,,${ }^{39, c}$ C. Hackstein, ${ }^{39}$ F. Hartmann, ${ }^{39, c}$ T. Hauth, ${ }^{39, c}$ M. Heinrich,${ }^{39}$ H. Held, ${ }^{39}$ K. H. Hoffmann, ${ }^{39}$ U. Husemann, ${ }^{39}$ I. Katkov, ${ }^{39, q}$ J. R. Komaragiri, ${ }^{39}$ P. Lobelle Pardo,${ }^{39}$ D. Martschei, ${ }^{39}$ S. Mueller, ${ }^{39}$ Th. Müller, ${ }^{39}$ M. Niegel, ${ }^{39}$ A. Nürnberg, ${ }^{39}$ O. Oberst, ${ }^{39}$ A. Oehler, ${ }^{39}$ J. Ott, ${ }^{39}$ G. Quast,${ }^{39}$ K. Rabbertz,${ }^{39}$ F. Ratnikov, ${ }^{39}$ N. Ratnikova, ${ }^{39}$ S. Röcker, ${ }^{39}$

F.-P. Schilling, ${ }^{39}$ G. Schott, ${ }^{39}$ H. J. Simonis, ${ }^{39}$ F. M. Stober, ${ }^{39}$ D. Troendle, ${ }^{39}$ R. Ulrich,,${ }^{39}$ J. Wagner-Kuhr, ${ }^{39}$ S. Wayand, ${ }^{39}$ T. Weiler, ${ }^{39}$ M. Zeise, ${ }^{39}$ G. Anagnostou, ${ }^{40}$ G. Daskalakis, ${ }^{40}$ T. Geralis ${ }^{40}$ S. Kesisoglou, ${ }^{40}$ A. Kyriakis, ${ }^{40}$

D. Loukas, ${ }^{40}$ I. Manolakos, ${ }^{40}$ A. Markou, ${ }^{40}$ C. Markou, ${ }^{40}$ E. Ntomari, ${ }^{40}$ L. Gouskos, ${ }^{41}$ T. J. Mertzimekis, ${ }^{41}$

A. Panagiotou, ${ }^{41}$ N. Saoulidou, ${ }^{41}$ I. Evangelou, ${ }^{42}$ C. Foudas, ${ }^{42}$ P. Kokkas, ${ }^{42}$ N. Manthos, ${ }^{42}$ I. Papadopoulos,${ }^{42}$ V. Patras, ${ }^{42}$ G. Bencze, ${ }^{43}$ C. Hajdu,${ }^{43}$ P. Hidas,${ }^{43}$ D. Horvath,${ }^{43, t}$ F. Sikler, ${ }^{43}$ V. Veszpremi, ${ }^{43}$ G. Vesztergombi ${ }^{43, u}$ N. Beni,${ }^{44}$ S. Czellar, ${ }^{44}$ J. Molnar, ${ }^{44}$ J. Palinkas, ${ }^{44}$ Z. Szillasi, ${ }^{44}$ J. Karancsi, ${ }^{45}$ P. Raics,${ }^{45}$ Z. L. Trocsanyi, ${ }^{45}$ B. Ujvari, ${ }^{45}$ S. B. Beri, ${ }^{46}$ V. Bhatnagar, ${ }^{46}$ N. Dhingra, ${ }^{46}$ R. Gupta, ${ }^{46}$ M. Kaur, ${ }^{46}$ M. Z. Mehta, ${ }^{46}$ N. Nishu ${ }^{46}$ L. K. Saini,${ }^{46}$ A. Sharma, ${ }^{46}$ J. B. Singh, ${ }^{46}$ Ashok Kumar, ${ }^{47}$ Arun Kumar, ${ }^{47}$ S. Ahuja ${ }^{47}$ A. Bhardwaj, ${ }^{47}$ B. C. Choudhary, ${ }^{47}$ S. Malhotra, ${ }^{47}$ M. Naimuddin, ${ }^{47}$ K. Ranjan, ${ }^{47}$ V. Sharma,${ }^{47}$ R. K. Shivpuri, ${ }^{47}$ S. Banerjee, ${ }^{48}$ S. Bhattacharya, ${ }^{48}$ S. Dutta, ${ }^{48}$ B. Gomber, ${ }^{48}$ Sa. Jain ${ }^{48}$ Sh. Jain,${ }^{48}$ R. Khurana, ${ }^{48}$ S. Sarkar ${ }^{48}$ M. Sharan,${ }^{48}$ A. Abdulsalam, ${ }^{49}$ D. Dutta, ${ }^{49}$ S. Kailas, ${ }^{49}$ V. Kumar, ${ }^{49}$ A. K. Mohanty, ${ }^{49, c}$ L. M. Pant, ${ }^{49}$ P. Shukla, ${ }^{49}$ T. Aziz,${ }^{50}$ S. Ganguly, ${ }^{50}$ M. Guchait ${ }^{50, v}$ A. Gurtu,${ }^{50, w}$ M. Maity, ${ }^{50, x}$ G. Majumder, ${ }^{50}$ K. Mazumdar, ${ }^{50}$ G. B. Mohanty, ${ }^{50}$ B. Parida, ${ }^{50}$ K. Sudhakar,${ }^{50}$ N. Wickramage, ${ }^{50}$ S. Banerjee, ${ }^{51}$ S. Dugad ${ }^{51}$ H. Arfaei, ${ }^{52, y}$ H. Bakhshiansohi, ${ }^{52}$ S. M. Etesami, ${ }^{52, z}$ A. Fahim,${ }^{52, y}$ M. Hashemi, ${ }^{52, a a}$ H. Hesari, ${ }^{52}$ A. Jafari, ${ }^{52}$ M. Khakzad, ${ }^{52}$ M. Mohammadi Najafabadi, ${ }^{52}$ S. Paktinat Mehdiabadi, ${ }^{52}$ B. Safarzadeh, ${ }^{52, b b}$ M. Zeinali, ${ }^{52}$ M. Abbrescia, ${ }^{53 a, 53 b}$ 
L. Barbone, ${ }^{53 a, 53 b}$ C. Calabria, ${ }^{53 a, 53 b, c}$ S. S. Chhibra ${ }^{53 a, 53 b}$ A. Colaleo, ${ }^{53 a}$ D. Creanza, ${ }^{53 a, 53 c}$ N. De Filippis, ${ }^{53 a, 53 c, c}$ M. De Palma, ${ }^{53 a, 53 b}$ L. Fiore, ${ }^{53 a}$ G. Iaselli, ${ }^{53 a, 53 c}$ G. Maggi, ${ }^{53 a, 53 c}$ M. Maggi, ${ }^{53 a}$ B. Marangelli, ${ }^{53 a, 53 b}$ S. My, ${ }^{53 a, 53 c}$ S. Nuzzo, ${ }^{53 a, 53 b}$ N. Pacifico, ${ }^{53 a}$ A. Pompili, ${ }^{53 a, 53 b}$ G. Pugliese, ${ }^{53 a, 53 c}$ G. Selvaggi, ${ }^{53 a, 53 b}$ L. Silvestris, ${ }^{53 a}$

G. Singh, ${ }^{53 a, 53 b}$ R. Venditti, ${ }^{53 a, 53 b}$ P. Verwilligen, ${ }^{53 a}$ G. Zito, ${ }^{53 a}$ G. Abbiendi, ${ }^{54 a}$ A. C. Benvenuti, ${ }^{54 a}$ D. Bonacorsi, ${ }^{54 a, 54 b}$ S. Braibant-Giacomelli, ${ }^{54 a, 54 b}$ L. Brigliadori, ${ }^{54 a, 54 b}$ P. Capiluppi, ${ }^{54 a, 54 b}$ A. Castro, ${ }^{54 a, 54 b}$ F. R. Cavallo, ${ }^{54 a}$ M. Cuffiani, ${ }^{54 a, 54 b}$ G. M. Dallavalle, ${ }^{54 a}$ F. Fabbri, ${ }^{54 a}$ A. Fanfani, ${ }^{54 a, 54 b}$ D. Fasanella, ${ }^{54 a, 54 b}$ P. Giacomelli, ${ }^{54 a}$ C. Grandi, ${ }^{54 a}$ L. Guiducci, ${ }^{54 a, 54 b}$ S. Marcellini, ${ }^{54 a}$ G. Masetti, ${ }^{54 a}$ M. Meneghelli, ${ }^{54 a, 54 b, c}$ A. Montanari, ${ }^{54 a}$ F. L. Navarria, ${ }^{54 a, 54 b}$ F. Odorici, ${ }^{54 a}$ A. Perrotta, ${ }^{54 a}$ F. Primavera, ${ }^{54 a, 54 b}$ A. M. Rossi, ${ }^{54 a, 54 b}$ T. Rovelli, ${ }^{54 a, 54 b}$ G. P. Siroli, ${ }^{54 a, 54 b}$ N. Tosi, ${ }^{54 a}$ R. Travaglini, ${ }^{54 a, 54 b}$ S. Albergo, ${ }^{55 a, 55 b}$ G. Cappello, ${ }^{55 a, 55 b}$ M. Chiorboli, ${ }^{55 a, 55 b}$ S. Costa, ${ }^{55 a, 55 b}$ R. Potenza ${ }^{55 a, 55 b}$ A. Tricomi, ${ }^{55 a, 55 b}$ C. Tuve,${ }^{55 a, 55 b}$ G. Barbagli, ${ }^{56 a}$ V. Ciulli, ${ }^{56,56 b}$ C. Civinini, ${ }^{56 a}$ R. D'Alessandro, ${ }^{56 a, 56 b}$ E. Focardi, ${ }^{56 a, 56 b}$ S. Frosali, ${ }^{56 a, 56 b}$ E. Gallo, ${ }^{56 a}$ S. Gonzi, ${ }^{56 a, 56 b}$ M. Meschini, ${ }^{56 a}$ S. Paoletti, ${ }^{56 a}$ G. Sguazzoni, ${ }^{56 a}$ A. Tropiano, ${ }^{56 a, 56 b}$ L. Benussi ${ }^{57}$ S. Bianco, ${ }^{57}$ S. Colafranceschi,${ }^{57, c c}$ F. Fabbri, ${ }^{57}$ D. Piccolo, ${ }^{57}$ P. Fabbricatore, ${ }^{58 a}$ R. Musenich,${ }^{58 a}$ S. Tosi, ${ }^{58 a, 58 b}$ A. Benaglia, ${ }^{59 a}$ F. De Guio, ${ }^{59 a, 59 b}$ L. Di Matteo, ${ }^{59 a, 59 b, c}$ S. Fiorendi, ${ }^{59 a, 59 b}$ S. Gennai, ${ }^{59 a, c}$ A. Ghezzi, ${ }^{59 a, 59 b}$ S. Malvezzi, ${ }^{59 a}$ R. A. Manzoni, ${ }^{59 a, 59 b}$ A. Martelli, ${ }^{59 a, 59 b}$ A. Massironi, ${ }^{59 a, 59 b}$ D. Menasce, ${ }^{59 a}$ L. Moroni, ${ }^{59 a}$ M. Paganoni, ${ }^{59 a, 59 b}$ D. Pedrini, ${ }^{59 a}$ S. Ragazzi, ${ }^{59 a, 59 b}$ N. Redaelli, ${ }^{59 a}$ S. Sala, ${ }^{59 a}$ T. Tabarelli de Fatis, ${ }^{59 a, 59 b}$ S. Buontempo, ${ }^{60 a}$ C. A. Carrillo Montoya, ${ }^{60 a}$ N. Cavallo, ${ }^{60 a, 60 c}$ A. De Cosa,${ }^{60 a, 60 b, c}$ O. Dogangun, ${ }^{60 a, 60 b}$ F. Fabozzi, ${ }^{60 a, 60 c}$ A. O. M. Iorio, ${ }^{60 a, 60 b}$ L. Lista, ${ }^{60 a}$ S. Meola, ${ }^{60 a, 60 d, d d}$ M. Merola, ${ }^{60 a}$ P. Paolucci, ${ }^{60 a, c}$ P. Azzi, ${ }^{61 a}$ N. Bacchetta, ${ }^{61 a, c}$ D. Bisello, ${ }^{61 a, 61 b}$ A. Branca, ${ }^{61 a, 61 b, c}$ R. Carlin, ${ }^{61 \mathrm{a}, 61 \mathrm{~b}}$ P. Checchia, ${ }^{61 \mathrm{a}}$ T. Dorigo ${ }^{61 \mathrm{a}}$ F. Gasparini, ${ }^{61 \mathrm{a}, 61 \mathrm{~b}}$ U. Gasparini, ${ }^{61 \mathrm{a}, 61 \mathrm{~b}}$ A. Gozzelino, ${ }^{61 \mathrm{a}}$ K. Kanishchev, ${ }^{61 a, 61 \mathrm{c}}$ S. Lacaprara, ${ }^{61 \mathrm{a}}$ I. Lazzizzera, ${ }^{61 \mathrm{a}, 61 \mathrm{c}}$ M. Margoni ${ }^{61 \mathrm{a}, 61 \mathrm{~b}}$ A. T. Meneguzzo, ${ }^{61 \mathrm{a}, 61 \mathrm{~b}}$ J. Pazzini, ${ }^{61 \mathrm{a}, 61 \mathrm{~b}}$ N. Pozzobon, ${ }^{61 \mathrm{a}, 61 \mathrm{~b}}$ P. Ronchese, ${ }^{61 \mathrm{a}, 61 \mathrm{~b}}$ F. Simonetto, ${ }^{61 \mathrm{a}, 61 \mathrm{~b}}$ E. Torassa, ${ }^{61 \mathrm{a}}$ M. Tosi, ${ }^{61 \mathrm{a}, 61 \mathrm{~b}}$ S. Vanini ${ }^{61 \mathrm{a}, 61 \mathrm{~b}}$ P. Zotto, ${ }^{61 \mathrm{a}, 61 \mathrm{~b}}$ A. Zucchetta, ${ }^{61 \mathrm{a}, 61 \mathrm{~b}}$ G. Zumerle, ${ }^{61 \mathrm{a}, 61 \mathrm{~b}}$ M. Gabusi, ${ }^{62 \mathrm{a}, 62 \mathrm{~b}}$ S. P. Ratti, ${ }^{62 \mathrm{a}, 62 \mathrm{~b}}$ C. Riccardi, ${ }^{62 a, 62 b}$ P. Torre, ${ }^{62 a, 62 b}$ P. Vitulo, ${ }^{62 a, 62 b}$ M. Biasini, ${ }^{63 a, 63 b}$ G. M. Bilei, ${ }^{63 a}$ L. Fanò, ${ }^{63 a, 63 b}$ P. Lariccia, ${ }^{63 a, 63 b}$ G. Mantovani, ${ }^{63 a, 63 b}$ M. Menichelli, ${ }^{63 a}$ A. Nappi, ${ }^{63 a, 63 b, a}$ F. Romeo, ${ }^{63 a, 63 b}$ A. Saha, ${ }^{63 a}$ A. Santocchia, ${ }^{63 a, 63 b}$ A. Spiezia, ${ }^{63 a, 63 b}$ S. Taroni, ${ }^{63 a, 63 b}$ P. Azzurri, ${ }^{64 a, 64 c}$ G. Bagliesi, ${ }^{64 a}$ J. Bernardini, ${ }^{64 a}$ T. Boccali, ${ }^{64 a}$ G. Broccolo, ${ }^{64 a, 64 c}$ R. Castaldi, ${ }^{64 a}$ R. T. D’Agnolo, ${ }^{64 a, 64 c, c}$ R. Dell'Orso, ${ }^{64 a}$ F. Fiori, ${ }^{64 a, 64 b, c}$ L. Foà ${ }^{64 a, 64 c}$ A. Giassi, ${ }^{64 a}$ A. Kraan ${ }^{64 a}$ F. Ligabue ${ }^{64 a, 64 c}$ T. Lomtadze, ${ }^{64 a}$ L. Martini,${ }^{64 a, e e}$ A. Messineo ${ }^{64 a, 64 b}$ F. Palla, ${ }^{64 a}$ A. Rizzi, ${ }^{64 a, 64 b}$ A. T. Serban, ${ }^{64 a, f f}$ P. Spagnolo, ${ }^{64 a}$ P. Squillacioti, ${ }^{64 a, c}$ R. Tenchini, ${ }^{64 a}$ G. Tonelli, ${ }^{64 a, 64 b}$ A. Venturi, ${ }^{64 a}$ P. G. Verdini, ${ }^{64 a}$ L. Barone, ${ }^{65 a, 65 b}$ F. Cavallari, ${ }^{65 a}$ D. Del Re,${ }^{65,65 b}$ M. Diemoz, ${ }^{65 a}$ C. Fanelli, ${ }^{65 a, 65 b}$ M. Grassi, ${ }^{65 a, 65 b, c}$ E. Longo, ${ }^{65 a, 65 b}$ P. Meridiani, ${ }^{65 a, c}$ F. Micheli, ${ }^{65 a, 65 b}$ S. Nourbakhsh ${ }^{65 a, 65 b}$ G. Organtini, ${ }^{65 a, 65 b}$ R. Paramatti, ${ }^{65 a}$ S. Rahatlou, ${ }^{65 a, 65 b}$ M. Sigamani, ${ }^{65 a}$

L. Soffi, ${ }^{65 a, 65 b}$ N. Amapane, ${ }^{66 a, 66 b}$ R. Arcidiacono, ${ }^{66 a, 66 c}$ S. Argiro, ${ }^{66 a, 66 b}$ M. Arneodo, ${ }^{66 a, 66 c}$ C. Biino, ${ }^{66 a}$ N. Cartiglia, ${ }^{66 a}$ S. Casasso, ${ }^{66 a, 66 b}$ M. Costa, ${ }^{66 a, 66 b}$ N. Demaria, ${ }^{66 a}$ C. Mariotti, ${ }^{66 a, c}$ S. Maselli, ${ }^{66 a}$ E. Migliore, ${ }^{66 a, 66 b}$ V. Monaco, ${ }^{66 a, 66 b}$ M. Musich, ${ }^{66 a, c}$ M. M. Obertino, ${ }^{66 a, 66 c}$ N. Pastrone, ${ }^{66 a}$ M. Pelliccioni ${ }^{66 a}$ A. Potenza, ${ }^{66 a, 66 b}$ A. Romero, ${ }^{66 a, 66 b}$ M. Ruspa, ${ }^{66 a, 66 c}$ R. Sacchi, ${ }^{66 a, 66 b}$ A. Solano, ${ }^{66 a, 66 b}$ A. Staiano, ${ }^{66 a}$ S. Belforte, ${ }^{67 a}$ V. Candelise, ${ }^{67 a, 67 b}$ M. Casarsa, ${ }^{67 a}$ F. Cossutti, ${ }^{67 a}$ G. Della Ricca, ${ }^{67 a, 67 b}$ B. Gobbo, ${ }^{67 a}$ M. Marone, ${ }^{67 a, 67 b, c}$ D. Montanino, ${ }^{67 a, 67 b, c}$ A. Penzo, ${ }^{67 a}$ A. Schizzi, ${ }^{67 a, 67 b}$ T. Y. Kim, ${ }^{68}$ S. K. Nam, ${ }^{68}$ S. Chang, ${ }^{69}$ D. H. Kim, ${ }^{69}$ G. N. Kim, ${ }^{69}$ D. J. Kong, ${ }^{69}$ H. Park, ${ }^{69}$ D. C. Son, ${ }^{69}$ T. Son, ${ }^{69}$ J. Y. Kim, ${ }^{70}$ Zero J. Kim, ${ }^{70}$ S. Song, ${ }^{70}$ S. Choi, ${ }^{71}$ D. Gyun, ${ }^{71}$ B. Hong, ${ }^{71}$ M. Jo, ${ }^{71}$ H. Kim, ${ }^{71}$ T. J. Kim,${ }^{71}$ K. S. Lee, ${ }^{71}$ D. H. Moon,${ }^{71}$ S. K. Park, ${ }^{71}$ Y. Roh, ${ }^{71}$ M. Choi, ${ }^{72}$ J. H. Kim,${ }^{72}$ C. Park, ${ }^{72}$ I. C. Park, ${ }^{72}$ S. Park, ${ }^{72}$ G. Ryu, ${ }^{72}$ Y. Choi, ${ }^{73}$ Y. K. Choi,${ }^{73}$ J. Goh,${ }^{73}$ M. S. Kim, ${ }^{73}$ E. Kwon, ${ }^{73}$ B. Lee, ${ }^{73}$ J. Lee ${ }^{73}$ S. Lee ${ }^{73}$ H. Seo,${ }^{73}$ I. Yu ${ }^{73}$ M. J. Bilinskas, ${ }^{74}$ I. Grigelionis,${ }^{74}$ M. Janulis, ${ }^{74}$ A. Juodagalvis, ${ }^{74}$ H. Castilla-Valdez, ${ }^{75}$ E. De La Cruz-Burelo, ${ }^{75}$ I. Heredia-de La Cruz, ${ }^{75}$ R. Lopez-Fernandez,${ }^{75}$ J. Martínez-Ortega, ${ }^{75}$ A. Sanchez-Hernandez, ${ }^{75}$ L. M. Villasenor-Cendejas, ${ }^{75}$ S. Carrillo Moreno, ${ }^{76}$ F. Vazquez Valencia, ${ }^{76}$ H. A. Salazar Ibarguen, ${ }^{77}$ E. Casimiro Linares, ${ }^{78}$ A. Morelos Pineda ${ }^{78}$ M. A. Reyes-Santos, ${ }^{78}$ D. Krofcheck,${ }^{79}$ A. J. Bell, ${ }^{80}$ P. H. Butler, ${ }^{80}$ R. Doesburg, ${ }^{80}$ S. Reucroft, ${ }^{80}$ H. Silverwood, ${ }^{80}$ M. Ahmad ${ }^{81}$ M. I. Asghar, ${ }^{81}$ J. Butt, ${ }^{81}$ H. R. Hoorani, ${ }^{81}$ S. Khalid, ${ }^{81}$ W. A. Khan ${ }^{81}$ T. Khurshid, ${ }^{81}$ S. Qazi, ${ }^{81}$ M. A. Shah, ${ }^{81}$ M. Shoaib ${ }^{81}$ H. Bialkowska, ${ }^{82}$ B. Boimska, ${ }^{82}$ T. Frueboes, ${ }^{82}$ M. Górski, ${ }^{82}$ M. Kazana, ${ }^{82}$ K. Nawrocki, ${ }^{82}$ K. Romanowska-Rybinska, ${ }^{82}$ M. Szleper, ${ }^{82}$ G. Wrochna, ${ }^{82}$ P. Zalewski, ${ }^{82}$ G. Brona,${ }^{83}$ K. Bunkowski, ${ }^{83}$ M. Cwiok, ${ }^{83}$ W. Dominik, ${ }^{83}$ K. Doroba, ${ }^{83}$

A. Kalinowski, ${ }^{83}$ M. Konecki, ${ }^{83}$ J. Krolikowski, ${ }^{83}$ M. Misiura, ${ }^{83}$ N. Almeida ${ }^{84}$ P. Bargassa, ${ }^{84}$ A. David ${ }^{84}$ P. Faccioli, ${ }^{84}$ P. G. Ferreira Parracho,${ }^{84}$ M. Gallinaro, ${ }^{84}$ J. Seixas, ${ }^{84}$ J. Varela ${ }^{84}$ P. Vischia, ${ }^{84}$ I. Belotelov,${ }^{85}$ P. Bunin, ${ }^{85}$ I. Golutvin, ${ }^{85}$ I. Gorbunov, ${ }^{85}$ A. Kamenev, ${ }^{85}$ V. Karjavin, ${ }^{85}$ G. Kozlov, ${ }^{85}$ A. Lanev, ${ }^{85}$ A. Malakhov, ${ }^{85}$ P. Moisenz,${ }^{85}$ V. Palichik, ${ }^{85}$ V. Perelygin, ${ }^{85}$ M. Savina,${ }^{85}$ S. Shmatov, ${ }^{85}$ V. Smirnov, ${ }^{85}$ A. Volodko, ${ }^{85}$ A. Zarubin, ${ }^{85}$ S. Evstyukhin, ${ }^{86}$ 
V. Golovtsov, ${ }^{86}$ Y. Ivanov, ${ }^{86}$ V. Kim, ${ }^{86}$ P. Levchenko, ${ }^{86}$ V. Murzin, ${ }^{86}$ V. Oreshkin, ${ }^{86}$ I. Smirnov, ${ }^{86}$ V. Sulimov, ${ }^{86}$ L. Uvarov ${ }^{86}$ S. Vavilov, ${ }^{86}$ A. Vorobyev, ${ }^{86}$ An. Vorobyev, ${ }^{86}$ Yu. Andreev, ${ }^{87}$ A. Dermenev, ${ }^{87}$ S. Gninenko, ${ }^{87}$ N. Golubev, ${ }^{87}$ M. Kirsanov, ${ }^{87}$ N. Krasnikov, ${ }^{87}$ V. Matveev, ${ }^{87}$ A. Pashenkov, ${ }^{87}$ D. Tlisov, ${ }^{87}$ A. Toropin, ${ }^{87}$ V. Epshteyn, ${ }^{88}$ M. Erofeeva, ${ }^{88}$ V. Gavrilov, ${ }^{88}$ M. Kossov, ${ }^{88}$ N. Lychkovskaya, ${ }^{88}$ V. Popov, ${ }^{88}$ G. Safronov, ${ }^{88}$ S. Semenov, ${ }^{88}$ I. Shreyber, ${ }^{88}$ V. Stolin,${ }^{88}$ E. Vlasov, ${ }^{88}$ A. Zhokin, ${ }^{88}$ A. Belyaev,${ }^{89}$ E. Boos, ${ }^{89}$ M. Dubinin, ${ }^{89, e}$ L. Dudko, ${ }^{89}$ A. Ershov, ${ }^{89}$ A. Gribushin, ${ }^{89}$ V. Klyukhin, ${ }^{89}$ O. Kodolova, ${ }^{89}$ I. Lokhtin, ${ }^{89}$ A. Markina, ${ }^{89}$ S. Obraztsov, ${ }^{89}$ M. Perfilov, ${ }^{89}$ S. Petrushanko, ${ }^{89}$ A. Popov, ${ }^{89}$ L. Sarycheva, ${ }^{89, \text { a }}$ V. Savrin, ${ }^{89}$ A. Snigirev, ${ }^{89}$ V. Andreev, ${ }^{90}$ M. Azarkin,${ }^{90}$ I. Dremin, ${ }^{90}$ M. Kirakosyan, ${ }^{90}$ A. Leonidov, ${ }^{90}$ G. Mesyats, ${ }^{90}$ S. V. Rusakov, ${ }^{90}$ A. Vinogradov, ${ }^{90}$ I. Azhgirey, ${ }^{91}$

I. Bayshev, ${ }^{91}$ S. Bitioukov, ${ }^{91}$ V. Grishin, ${ }^{91, \mathrm{c}}$ V. Kachanov, ${ }^{91}$ D. Konstantinov,${ }^{91}$ V. Krychkine, ${ }^{91}$ V. Petrov, ${ }^{91}$ R. Ryutin, ${ }^{91}$ A. Sobol, ${ }^{91}$ L. Tourtchanovitch, ${ }^{91}$ S. Troshin,,${ }^{91}$ N. Tyurin,,${ }^{91}$ A. Uzunian, ${ }^{91}$ A. Volkov, ${ }^{91}$ P. Adzic, ${ }^{92, g g}$ M. Djordjevic, ${ }^{92}$ M. Ekmedzic, ${ }^{92}$ D. Krpic, ${ }^{92, g 9}$ J. Milosevic, ${ }^{92}$ M. Aguilar-Benitez, ${ }^{93}$ J. Alcaraz Maestre,${ }^{93}$

P. Arce, ${ }^{93}$ C. Battilana, ${ }^{93}$ E. Calvo, ${ }^{93}$ M. Cerrada,${ }^{93}$ M. Chamizo Llatas,${ }^{93}$ N. Colino, ${ }^{93}$ B. De La Cruz,${ }^{93}$ A. Delgado Peris, ${ }^{93}$ D. Domínguez Vázquez, ${ }^{93}$ C. Fernandez Bedoya, ${ }^{93}$ J. P. Fernández Ramos, ${ }^{93}$ A. Ferrando, ${ }^{93}$ J. Flix, ${ }^{93}$ M. C. Fouz, ${ }^{93}$ P. Garcia-Abia, ${ }^{93}$ O. Gonzalez Lopez, ${ }^{93}$ S. Goy Lopez,${ }^{93}$ J. M. Hernandez ${ }^{93}$ M. I. Josa, ${ }^{93}$ G. Merino, ${ }^{93}$ J. Puerta Pelayo, ${ }^{93}$ A. Quintario Olmeda,${ }^{93}$ I. Redondo, ${ }^{93}$ L. Romero, ${ }^{93}$ J. Santaolalla, ${ }^{93}$ M. S. Soares, ${ }^{93}$ C. Willmott, ${ }^{93}$ C. Albajar, ${ }^{94}$ G. Codispoti, ${ }^{94}$ J. F. de Trocóniz, ${ }^{94}$ H. Brun, ${ }^{95}$ J. Cuevas, ${ }^{95}$ J. Fernandez Menendez, ${ }^{95}$ S. Folgueras, ${ }^{95}$ I. Gonzalez Caballero, ${ }^{95}$ L. Lloret Iglesias, ${ }^{95}$ J. Piedra Gomez, ${ }^{95}$ J. A. Brochero Cifuentes, ${ }^{96}$ I. J. Cabrillo, ${ }^{96}$ A. Calderon, ${ }^{96}$ S. H. Chuang, ${ }^{96}$ J. Duarte Campderros,${ }^{96}$ M. Felcini,${ }^{96, \text { hh }}$ M. Fernandez, ${ }^{96}$ G. Gomez, ${ }^{96}$ J. Gonzalez Sanchez,${ }^{96}$ A. Graziano, ${ }^{96}$ C. Jorda, ${ }^{96}$ A. Lopez Virto, ${ }^{96}$ J. Marco, ${ }^{96}$ R. Marco,${ }^{96}$ C. Martinez Rivero, ${ }^{96}$ F. Matorras, ${ }^{96}$ F. J. Munoz Sanchez, ${ }^{96}$ T. Rodrigo, ${ }^{96}$ A. Y. Rodríguez-Marrero, ${ }^{96}$

A. Ruiz-Jimeno, ${ }^{96}$ L. Scodellaro, ${ }^{96}$ I. Vila, ${ }^{96}$ R. Vilar Cortabitarte, ${ }^{96}$ D. Abbaneo, ${ }^{97}$ E. Auffray, ${ }^{97}$ G. Auzinger,${ }^{97}$ M. Bachtis, ${ }^{97}$ P. Baillon, ${ }^{97}$ A. H. Ball, ${ }^{97}$ D. Barney, ${ }^{97}$ J. F. Benitez, ${ }^{97}$ C. Bernet, ${ }^{97,}$, G. Bianchi, ${ }^{97}$ P. Bloch,${ }^{97}$ A. Bocci, ${ }^{97}$ A. Bonato, ${ }^{97}$ C. Botta, ${ }^{97}$ H. Breuker, ${ }^{97}$ T. Camporesi,${ }^{97}$ G. Cerminara, ${ }^{97}$ T. Christiansen, ${ }^{97}$ J. A. Coarasa Perez, ${ }^{97}$ D. D'Enterria, ${ }^{97}$ A. Dabrowski, ${ }^{97}$ A. De Roeck,${ }^{97}$ S. Di Guida, ${ }^{97}$ M. Dobson, ${ }^{97}$ N. Dupont-Sagorin, ${ }^{97}$ A. Elliott-Peisert, ${ }^{97}$ B. Frisch, ${ }^{97}$ W. Funk, ${ }^{97}$ G. Georgiou, ${ }^{97}$ M. Giffels, ${ }^{97}$ D. Gigi, ${ }^{97}$ K. Gill ${ }^{97}$ D. Giordano,${ }^{97}$ M. Girone,${ }^{97}$ M. Giunta, ${ }^{97}$ F. Glege, ${ }^{97}$ R. Gomez-Reino Garrido,${ }^{97}$ P. Govoni, ${ }^{97}$ S. Gowdy, ${ }^{97}$ R. Guida, ${ }^{97}$ S. Gundacker, ${ }^{97}$ J. Hammer,${ }^{97}$ M. Hansen, ${ }^{97}$ P. Harris,${ }^{97}$ C. Hartl, ${ }^{97}$ J. Harvey, ${ }^{97}$ B. Hegner, ${ }^{97}$ A. Hinzmann, ${ }^{97}$ V. Innocente, ${ }^{97}$ P. Janot,,${ }^{97}$ K. Kaadze, ${ }^{97}$ E. Karavakis, ${ }^{97}$ K. Kousouris, ${ }^{97}$ P. Lecoq, ${ }^{97}$ Y.-J. Lee, ${ }^{97}$ P. Lenzi, ${ }^{97}$ C. Lourenço, ${ }^{97}$ N. Magini, ${ }^{97}$ T. Mäki, ${ }^{97}$ M. Malberti, ${ }^{97}$ L. Malgeri, ${ }^{97}$ M. Mannelli, ${ }^{97}$ L. Masetti, ${ }^{97}$ F. Meijers, ${ }^{97}$ S. Mersi, ${ }^{97}$ E. Meschi, ${ }^{97}$ R. Moser,${ }^{97}$ M. U. Mozer, ${ }^{97}$ M. Mulders, ${ }^{97}$ P. Musella,${ }^{97}$ E. Nesvold, ${ }^{97}$ L. Orsini, ${ }^{97}$ E. Palencia Cortezon, ${ }^{97}$ E. Perez, ${ }^{97}$ L. Perrozzi, ${ }^{97}$ A. Petrilli, ${ }^{97}$ A. Pfeiffer, ${ }^{97}$ M. Pierini,,${ }^{97}$ M. Pimiä, ${ }^{97}$ D. Piparo, ${ }^{97}$ G. Polese, ${ }^{97}$ L. Quertenmont,${ }^{97}$ A. Racz, ${ }^{97}$ W. Reece, ${ }^{97}$ J. Rodrigues Antunes, ${ }^{97}$ G. Rolandi, ${ }^{97, i i}$ C. Rovelli,${ }^{97, j j}$ M. Rovere, ${ }^{97}$ H. Sakulin, ${ }^{97}$ F. Santanastasio, ${ }^{97}$ C. Schäfer, ${ }^{97}$ C. Schwick, ${ }^{97}$ I. Segoni,,${ }^{97}$ S. Sekmen, ${ }^{97}$ A. Sharma, ${ }^{97}$ P. Siegrist,${ }^{97}$ P. Silva, ${ }^{97}$ M. Simon, ${ }^{97}$ P. Sphicas,,${ }^{97, k k}$ D. Spiga,${ }^{97}$ A. Tsirou,${ }^{97}$ G. I. Veres, ${ }^{97, u}$ J. R. Vlimant, ${ }^{97}$ H. K. Wöhri, ${ }^{97}$ S. D. Worm,,${ }^{97,11}$ W. D. Zeuner, ${ }^{97}$ W. Bertl, ${ }^{98}$ K. Deiters,${ }^{98}$ W. Erdmann, ${ }^{98}$ K. Gabathuler, ${ }^{98}$ R. Horisberger, ${ }^{98}$ Q. Ingram, ${ }^{98}$ H. C. Kaestli, ${ }^{98}$ S. König,${ }^{98}$ D. Kotlinski, ${ }^{98}$ U. Langenegger, ${ }^{98}$ F. Meier, ${ }^{98}$ D. Renker, ${ }^{98}$ T. Rohe, ${ }^{98}$ L. Bäni, ${ }^{99}$ P. Bortignon, ${ }^{99}$ M. A. Buchmann, ${ }^{99}$ B. Casal, ${ }^{99}$ N. Chanon, ${ }^{99}$ A. Deisher, ${ }^{99}$ G. Dissertori, ${ }^{99}$ M. Dittmar, ${ }^{99}$ M. Donegà, ${ }^{99}$ M. Dünser, ${ }^{99}$ P. Eller, ${ }^{99}$ J. Eugster, ${ }^{99}$ K. Freudenreich, ${ }^{99}$ C. Grab, ${ }^{99}$ D. Hits, ${ }^{99}$ P. Lecomte, ${ }^{99}$ W. Lustermann, ${ }^{99}$ A. C. Marini, ${ }^{99}$ P. Martinez Ruiz del Arbol,${ }^{99}$ N. Mohr ${ }^{99}$ F. Moortgat, ${ }^{99}$ C. Nägeli, ${ }^{99, m m}$ P. Nef, ${ }^{99}$ F. Nessi-Tedaldi ${ }^{99}$ F. Pandolfi, ${ }^{99}$ L. Pape,${ }^{99}$ F. Pauss, ${ }^{99}$ M. Peruzzi, ${ }^{99}$ F. J. Ronga, ${ }^{99}$ M. Rossini, ${ }^{99}$ L. Sala, ${ }^{99}$ A. K. Sanchez ${ }^{99}$ A. Starodumov, ${ }^{99}$, nn B. Stieger, ${ }^{99}$ M. Takahashi, ${ }^{99}$ L. Tauscher, ${ }^{99, a}$ A. Thea, ${ }^{99}$ K. Theofilatos, ${ }^{99}$ D. Treille, ${ }^{99}$ C. Urscheler, ${ }^{99}$ R. Wallny, ${ }^{99}$ H. A. Weber, ${ }^{99}$ L. Wehrli, ${ }^{99}$ C. Amsler, ${ }^{100, \text { oo }}$ V. Chiochia, ${ }^{100}$ S. De Visscher, ${ }^{100}$ C. Favaro, ${ }^{100}$ M. Ivova Rikova, ${ }^{100}$ B. Kilminster,${ }^{100}$ B. Millan Mejias, ${ }^{100}$ P. Otiougova, ${ }^{100}$ P. Robmann, ${ }^{100}$ H. Snoek, ${ }^{100}$ S. Tupputi, ${ }^{100}$ M. Verzetti, ${ }^{100}$ Y. H. Chang, ${ }^{101}$ K. H. Chen, ${ }^{101}$ C. Ferro, ${ }^{101}$ C. M. Kuo, ${ }^{101}$ S. W. Li,${ }^{101}$ W. Lin, ${ }^{101}$ Y. J. Lu, ${ }^{101}$ A. P. Singh,${ }^{101}$ R. Volpe,${ }^{101}$ S. S. Yu, ${ }^{101}$ P. Bartalini, ${ }^{102}$ P. Chang, ${ }^{102}$ Y. H. Chang, ${ }^{102}$ Y. W. Chang,,${ }^{102}$ Y. Chao, ${ }^{102}$ K. F. Chen, ${ }^{102}$ C. Dietz, ${ }^{102}$ U. Grundler, ${ }^{102}$ W.-S. Hou, ${ }^{102}$ Y. Hsiung, ${ }^{102}$ K. Y. Kao ${ }^{102}$ Y. J. Lei, ${ }^{102}$ R.-S. Lu, ${ }^{102}$ D. Majumder, ${ }^{102}$ E. Petrakou, ${ }^{102}$ X. Shi, ${ }^{102}$ J. G. Shiu, ${ }^{102}$ Y. M. Tzeng, ${ }^{102}$ X. Wan,${ }^{102}$ M. Wang, ${ }^{102}$ B. Asavapibhop, ${ }^{103}$ N. Srimanobhas, ${ }^{103}$ A. Adiguzel, ${ }^{104}$

M. N. Bakirci, ${ }^{104, p p}$ S. Cerci, ${ }^{104, q q}$ C. Dozen, ${ }^{104}$ I. Dumanoglu, ${ }^{104}$ E. Eskut, ${ }^{104}$ S. Girgis,${ }^{104}$ G. Gokbulut, ${ }^{104}$ E. Gurpinar, ${ }^{104}$ I. Hos, ${ }^{104}$ E. E. Kangal, ${ }^{104}$ T. Karaman, ${ }^{104}$ G. Karapinar, ${ }^{104, r r}$ A. Kayis Topaksu, ${ }^{104}$ G. Onengut,${ }^{104}$ K. Ozdemir, ${ }^{104}$ S. Ozturk, ${ }^{104, \mathrm{ss}}$ A. Polatoz, ${ }^{104}$ K. Sogut,,${ }^{104, \mathrm{tt}}$ D. Sunar Cerci, ${ }^{104, \mathrm{qq}}$ B. Tali, ${ }^{104, \mathrm{qq}}$ H. Topakli, ${ }^{104, \mathrm{pp}}$ 
L. N. Vergili, ${ }^{104}$ M. Vergili, ${ }^{104}$ I. V. Akin, ${ }^{105}$ T. Aliev, ${ }^{105}$ B. Bilin, ${ }^{105}$ S. Bilmis,${ }^{105}$ M. Deniz, ${ }^{105}$ H. Gamsizkan, ${ }^{105}$ A. M. Guler, ${ }^{105}$ K. Ocalan, ${ }^{105}$ A. Ozpineci, ${ }^{105}$ M. Serin, ${ }^{105}$ R. Sever, ${ }^{105}$ U. E. Surat, ${ }^{105}$ M. Yalvac, ${ }^{105}$ E. Yildirim, ${ }^{105}$ M. Zeyrek, ${ }^{105}$ E. Gülmez, ${ }^{106}$ B. Isildak, ${ }^{106, \text { uu }}$ M. Kaya, ${ }^{106, v v}$ O. Kaya, ${ }^{106, v v}$ S. Ozkorucuklu, ${ }^{106, w w}$ N. Sonmez, ${ }^{106, x x}$ K. Cankocak, ${ }^{107}$ L. Levchuk, ${ }^{108}$ J. J. Brooke, ${ }^{109}$ E. Clement, ${ }^{109}$ D. Cussans, ${ }^{109}$ H. Flacher, ${ }^{109}$ R. Frazier, ${ }^{109}$ J. Goldstein, ${ }^{109}$ M. Grimes, ${ }^{109}$ G. P. Heath, ${ }^{109}$ H. F. Heath, ${ }^{109}$ L. Kreczko, ${ }^{109}$ S. Metson, ${ }^{109}$ D. M. Newbold, ${ }^{109,11}$ K. Nirunpong, ${ }^{109}$ A. Poll, ${ }^{109}$ S. Senkin, ${ }^{109}$ V. J. Smith, ${ }^{109}$ T. Williams, ${ }^{109}$ L. Basso, ${ }^{110, y y}$ K. W. Bell, ${ }^{10}$ A. Belyaev, ${ }^{110, y y}$ C. Brew, ${ }^{110}$ R. M. Brown, ${ }^{110}$ D. J. A. Cockerill, ${ }^{110}$ J. A. Coughlan, ${ }^{110}$ K. Harder,${ }^{10}$ S. Harper, ${ }^{110}$ J. Jackson, ${ }^{110}$ B. W. Kennedy, ${ }^{110}$ E. Olaiya, ${ }^{110}$ D. Petyt, ${ }^{110}$ B. C. Radburn-Smith,,${ }^{110}$

C. H. Shepherd-Themistocleous, ${ }^{110}$ I. R. Tomalin, ${ }^{110}$ W. J. Womersley, ${ }^{110}$ R. Bainbridge, ${ }^{111}$ G. Ball,,${ }^{11}$ R. Beuselinck, ${ }^{111}$ O. Buchmuller, ${ }^{111}$ D. Colling, ${ }^{111}$ N. Cripps,${ }^{111}$ M. Cutajar,${ }^{111}$ P. Dauncey,${ }^{111}$ G. Davies,${ }^{111}$ M. Della Negra, ${ }^{111}$ W. Ferguson, ${ }^{111}$ J. Fulcher,${ }^{11}$ D. Futyan, ${ }^{111}$ A. Gilbert, ${ }^{111}$ A. Guneratne Bryer, ${ }^{111}$ G. Hall, ${ }^{111}$ Z. Hatherell, ${ }^{111}$ J. Hays,${ }^{111}$ G. Iles,${ }^{111}$ M. Jarvis,${ }^{11}$ G. Karapostoli, ${ }^{111}$ L. Lyons, ${ }^{111}$ A.-M. Magnan, ${ }^{111}$ J. Marrouche, ${ }^{111}$ B. Mathias, ${ }^{111}$ R. Nandi, ${ }^{111}$ J. Nash, ${ }^{11}$ A. Nikitenko, ${ }^{111, \text { nn }}$ J. Pela, ${ }^{111}$ M. Pesaresi, ${ }^{111}$ K. Petridis, ${ }^{111}$ M. Pioppi ${ }^{111, z z}$ D. M. Raymond, ${ }^{111}$ S. Rogerson, ${ }^{111}$ A. Rose,${ }^{111}$ M. J. Ryan, ${ }^{111}$ C. Seez, ${ }^{111}$ P. Sharp, ${ }^{11, a}$ A. Sparrow, ${ }^{111}$ M. Stoye,${ }^{11}$ A. Tapper,${ }^{111}$ M. Vazquez Acosta, ${ }^{111}$ T. Virdee, ${ }^{111}$ S. Wakefield,${ }^{111}$ N. Wardle, ${ }^{111}$ T. Whyntie, ${ }^{111}$ M. Chadwick, ${ }^{112}$ J. E. Cole, ${ }^{112}$ P. R. Hobson, ${ }^{112}$ A. Khan, ${ }^{112}$ P. Kyberd, ${ }^{112}$ D. Leggat, ${ }^{112}$ D. Leslie, ${ }^{112}$

W. Martin, ${ }^{112}$ I. D. Reid, ${ }^{112}$ P. Symonds, ${ }^{112}$ L. Teodorescu, ${ }^{112}$ M. Turner,${ }^{112}$ K. Hatakeyama, ${ }^{113}$ H. Liu, ${ }^{113}$ T. Scarborough, ${ }^{113}$ O. Charaf, ${ }^{114}$ C. Henderson, ${ }^{114}$ P. Rumerio, ${ }^{114}$ A. Avetisyan, ${ }^{115}$ T. Bose, ${ }^{115}$ C. Fantasia, ${ }^{115}$ A. Heister, ${ }^{115}$ J. St. John, ${ }^{115}$ P. Lawson, ${ }^{115}$ D. Lazic, ${ }^{115}$ J. Rohlf, ${ }^{115}$ D. Sperka, ${ }^{115}$ L. Sulak, ${ }^{115}$ J. Alimena, ${ }^{116}$

S. Bhattacharya, ${ }^{116}$ G. Christopher, ${ }^{116}$ D. Cutts, ${ }^{116}$ Z. Demiragli, ${ }^{116}$ A. Ferapontov, ${ }^{116}$ A. Garabedian, ${ }^{116}$ U. Heintz, ${ }^{116}$ S. Jabeen, ${ }^{116}$ G. Kukartsev, ${ }^{116}$ E. Laird, ${ }^{116}$ G. Landsberg, ${ }^{116}$ M. Luk,${ }^{116}$ M. Narain, ${ }^{116}$ D. Nguyen, ${ }^{116}$ M. Segala, ${ }^{116}$ T. Sinthuprasith, ${ }^{116}$ T. Speer,${ }^{116}$ R. Breedon, ${ }^{117}$ G. Breto, ${ }^{117}$ M. Calderon De La Barca Sanchez, ${ }^{117}$ S. Chauhan, ${ }^{117}$ M. Chertok, ${ }^{117}$ J. Conway ${ }^{117}$ R. Conway, ${ }^{117}$ P. T. Cox,${ }^{117}$ J. Dolen, ${ }^{117}$ R. Erbacher, ${ }^{117}$ M. Gardner, ${ }^{117}$ R. Houtz, ${ }^{117}$ W. Ko,${ }^{117}$ A. Kopecky ${ }^{117}$ R. Lander, ${ }^{117}$ O. Mall, ${ }^{117}$ T. Miceli, ${ }^{17}$ D. Pellett, ${ }^{117}$ F. Ricci-Tam, ${ }^{117}$ B. Rutherford, ${ }^{117}$ M. Searle, ${ }^{117}$ J. Smith,${ }^{117}$ M. Squires, ${ }^{117}$ M. Tripathi, ${ }^{117}$ R. Vasquez Sierra, ${ }^{117}$ R. Yohay, ${ }^{117}$ V. Andreev, ${ }^{118}$ D. Cline, ${ }^{18}$ R. Cousins, ${ }^{18}$ J. Duris, ${ }^{118}$ S. Erhan, ${ }^{118}$ P. Everaerts, ${ }^{18}$ C. Farrell, ${ }^{118}$ J. Hauser, ${ }^{118}$ M. Ignatenko, ${ }^{118}$ C. Jarvis, ${ }^{118}$ G. Rakness, ${ }^{118}$ P. Schlein, ${ }^{118, a}$ P. Traczyk,${ }^{118}$ V. Valuev, ${ }^{118}$ M. Weber, ${ }^{118}$ J. Babb ${ }^{119}$ R. Clare, ${ }^{119}$ M.E. Dinardo, ${ }^{119}$ J. Ellison, ${ }^{119}$ J. W. Gary, ${ }^{119}$ F. Giordano, ${ }^{119}$ G. Hanson, ${ }^{119}$ H. Liu,${ }^{119}$ O. R. Long, ${ }^{119}$ A. Luthra, ${ }^{119}$ H. Nguyen, ${ }^{119}$ S. Paramesvaran, ${ }^{119}$ J. Sturdy, ${ }^{119}$ S. Sumowidagdo, ${ }^{119}$ R. Wilken,${ }^{119}$ S. Wimpenny, ${ }^{119}$

W. Andrews, ${ }^{120}$ J. G. Branson, ${ }^{120}$ G. B. Cerati, ${ }^{120}$ S. Cittolin, ${ }^{120}$ D. Evans, ${ }^{120}$ A. Holzner, ${ }^{120}$ R. Kelley, ${ }^{120}$

M. Lebourgeois,${ }^{120}$ J. Letts, ${ }^{120}$ I. Macneill, ${ }^{120}$ B. Mangano, ${ }^{120}$ S. Padhi,${ }^{120}$ C. Palmer ${ }^{120}$ G. Petrucciani, ${ }^{120}$ M. Pieri, ${ }^{120}$ M. Sani,${ }^{120}$ V. Sharma,${ }^{120}$ S. Simon, ${ }^{120}$ E. Sudano, ${ }^{120}$ M. Tadel, ${ }^{120}$ Y. Tu, ${ }^{120}$ A. Vartak,${ }^{120}$ S. Wasserbaech, ${ }^{120, \text { aaa }}$ F. Würthwein, ${ }^{120}$ A. Yagil, ${ }^{120}$ J. Yoo, ${ }^{120}$ D. Barge, ${ }^{121}$ R. Bellan, ${ }^{121}$ C. Campagnari, ${ }^{121}$ M. D’Alfonso, ${ }^{121}$ T. Danielson, ${ }^{121}$ K. Flowers, ${ }^{121}$ P. Geffert, ${ }^{121}$ F. Golf, ${ }^{121}$ J. Incandela, ${ }^{121}$ C. Justus, ${ }^{121}$ P. Kalavase, ${ }^{121}$ D. Kovalskyi, ${ }^{121}$ V. Krutelyov, ${ }^{121}$ S. Lowette, ${ }^{121}$ R. Magaña Villalba, ${ }^{121}$ N. Mccoll,,${ }^{121}$ V. Pavlunin, ${ }^{121}$ J. Ribnik, ${ }^{121}$ J. Richman, ${ }^{121}$ R. Rossin, ${ }^{121}$ D. Stuart, ${ }^{121}$ W. To, ${ }^{121}$ C. West, ${ }^{121}$ A. Apresyan, ${ }^{122}$ A. Bornheim, ${ }^{122}$ Y. Chen, ${ }^{122}$ E. Di Marco, ${ }^{122}$ J. Duarte, ${ }^{122}$ M. Gataullin, ${ }^{122}$ Y. Ma, ${ }^{122}$ A. Mott,,${ }^{122}$ H. B. Newman, ${ }^{122}$ C. Rogan, ${ }^{122}$ M. Spiropulu, ${ }^{122}$ V. Timciuc, ${ }^{122}$ J. Veverka, ${ }^{122}$ R. Wilkinson, ${ }^{122}$ S. Xie, ${ }^{122}$ Y. Yang, ${ }^{122}$ R. Y. Zhu, ${ }^{122}$ V. Azzolini, ${ }^{123}$ A. Calamba, ${ }^{123}$ R. Carroll, ${ }^{123}$ T. Ferguson, ${ }^{123}$ Y. Iiyama, ${ }^{123}$ D. W. Jang, ${ }^{123}$ Y. F. Liu, ${ }^{123}$ M. Paulini, ${ }^{123}$ H. Vogel, ${ }^{123}$ I. Vorobiev, ${ }^{123}$ J. P. Cumalat, ${ }^{124}$ B. R. Drell, ${ }^{124}$ W. T. Ford, ${ }^{124}$ A. Gaz,${ }^{124}$ E. Luiggi Lopez, ${ }^{124}$ J. G. Smith, ${ }^{124}$ K. Stenson, ${ }^{124}$ K. A. Ulmer, ${ }^{124}$ S. R. Wagner, ${ }^{124}$ J. Alexander, ${ }^{125}$ A. Chatterjee, ${ }^{125}$ N. Eggert, ${ }^{125}$ L. K. Gibbons, ${ }^{125}$ B. Heltsley, ${ }^{125}$ W. Hopkins, ${ }^{125}$ A. Khukhunaishvilii, ${ }^{125}$ B. Kreis, ${ }^{125}$ N. Mirman,${ }^{125}$ G. Nicolas Kaufman, ${ }^{125}$ J. R. Patterson, ${ }^{125}$ A. Ryd, ${ }^{125}$ E. Salvati, ${ }^{125}$ W. Sun, ${ }^{125}$ W. D. Teo, ${ }^{125}$ J. Thom, ${ }^{125}$ J. Thompson, ${ }^{125}$ J. Tucker, ${ }^{125}$ J. Vaughan, ${ }^{125}$ Y. Weng, ${ }^{125}$ L. Winstrom, ${ }^{125}$ P. Wittich, ${ }^{125}$ D. Winn,,${ }^{126}$ S. Abdullin, ${ }^{127}$ M. Albrow, ${ }^{127}$ J. Anderson, ${ }^{127}$ L. A. T. Bauerdick, ${ }^{127}$ A. Beretvas, ${ }^{127}$ J. Berryhill, ${ }^{127}$ P. C. Bhat, ${ }^{127}$ K. Burkett, ${ }^{127}$ J. N. Butler, ${ }^{127}$ V. Chetluru, ${ }^{127}$ H. W. K. Cheung, ${ }^{127}$ F. Chlebana, ${ }^{127}$ V. D. Elvira, ${ }^{127}$ I. Fisk, ${ }^{127}$ J. Freeman, ${ }^{127}$ Y. Gao, ${ }^{127}$ D. Green, ${ }^{127}$ O. Gutsche, ${ }^{127}$ J. Hanlon, ${ }^{127}$ R. M. Harris, ${ }^{127}$ J. Hirschauer, ${ }^{127}$ B. Hooberman, ${ }^{127}$ S. Jindariani, ${ }^{127}$ M. Johnson, ${ }^{127}$ U. Joshi, ${ }^{127}$ B. Klima, ${ }^{127}$ S. Kunori, ${ }^{127}$ S. Kwan, ${ }^{127}$ C. Leonidopoulos, ${ }^{127, b b b}$ J. Linacre, ${ }^{127}$ D. Lincoln, ${ }^{127}$ R. Lipton, ${ }^{127}$ J. Lykken, ${ }^{127}$ K. Maeshima, ${ }^{127}$ J. M. Marraffino, ${ }^{127}$ S. Maruyama, ${ }^{127}$ D. Mason, ${ }^{127}$ P. McBride,${ }^{127}$ K. Mishra, ${ }^{127}$ S. Mrenna, ${ }^{127}$ Y. Musienko, ${ }^{127, c c c}$ C. Newman-Holmes, ${ }^{127}$ V. O'Dell, ${ }^{127}$ O. Prokofyev, ${ }^{127}$ E. Sexton-Kennedy, ${ }^{127}$ S. Sharma, ${ }^{127}$ W. J. Spalding, ${ }^{127}$ L. Spiegel, ${ }^{127}$ L. Taylor, ${ }^{127}$ S. Tkaczyk,${ }^{127}$ N. V. Tran, ${ }^{127}$ L. Uplegger,${ }^{127}$ 
E. W. Vaandering, ${ }^{127}$ R. Vidal, ${ }^{127}$ J. Whitmore,${ }^{127}$ W. Wu ${ }^{127}$ F. Yang, ${ }^{127}$ J. C. Yun, ${ }^{127}$ D. Acosta, ${ }^{128}$ P. Avery, ${ }^{128}$ D. Bourilkov, ${ }^{128}$ M. Chen, ${ }^{128}$ T. Cheng, ${ }^{128}$ S. Das, ${ }^{128}$ M. De Gruttola ${ }^{128}$ G. P. Di Giovanni, ${ }^{128}$ D. Dobur, ${ }^{128}$ A. Drozdetskiy, ${ }^{128}$ R. D. Field, ${ }^{128}$ M. Fisher, ${ }^{128}$ Y. Fu, ${ }^{128}$ I. K. Furic, ${ }^{128}$ J. Gartner, ${ }^{128}$ J. Hugon, ${ }^{128}$ B. Kim, ${ }^{128}$ J. Konigsberg, ${ }^{128}$ A. Korytov, ${ }^{128}$ A. Kropivnitskaya, ${ }^{128}$ T. Kypreos, ${ }^{128}$ J. F. Low, ${ }^{128}$ K. Matchev, ${ }^{128}$ P. Milenovic, ${ }^{128, \text { ddd }}$ G. Mitselmakher, ${ }^{128}$ L. Muniz, ${ }^{128}$ M. Park, ${ }^{128}$ R. Remington, ${ }^{128}$ A. Rinkevicius, ${ }^{128}$ P. Sellers, ${ }^{128}$ N. Skhirtladze, ${ }^{128}$ M. Snowball, ${ }^{128}$ J. Yelton, ${ }^{128}$ M. Zakaria, ${ }^{128}$ V. Gaultney, ${ }^{129}$ S. Hewamanage, ${ }^{129}$ L. M. Lebolo, ${ }^{129}$ S. Linn, ${ }^{129}$ P. Markowitz, ${ }^{129}$ G. Martinez, ${ }^{129}$ J. L. Rodriguez, ${ }^{129}$ T. Adams, ${ }^{130}$ A. Askew, ${ }^{130}$ J. Bochenek, ${ }^{130}$ J. Chen, ${ }^{130}$ B. Diamond, ${ }^{130}$ S. V. Gleyzer, ${ }^{130}$ J. Haas, ${ }^{130}$ S. Hagopian,${ }^{130}$ V. Hagopian, ${ }^{130}$ M. Jenkins, ${ }^{130}$ K. F. Johnson, ${ }^{130}$ H. Prosper, ${ }^{130}$ V. Veeraraghavan, ${ }^{130}$ M. Weinberg, ${ }^{130}$ M. M. Baarmand, ${ }^{131}$ B. Dorney,${ }^{131}$ M. Hohlmann, ${ }^{131}$ H. Kalakhety, ${ }^{131}$ I. Vodopiyanov, ${ }^{131}$ F. Yumiceva, ${ }^{131}$ M. R. Adams, ${ }^{132}$ I. M. Anghel, ${ }^{132}$ L. Apanasevich ${ }^{132}$ Y. Bai, ${ }^{132}$ V.E. Bazterra, ${ }^{132}$ R. R. Betts, ${ }^{132}$ I. Bucinskaite, ${ }^{132}$ J. Callner, ${ }^{132}$ R. Cavanaugh, ${ }^{132}$ O. Evdokimov, ${ }^{132}$ L. Gauthier, ${ }^{132}$ C. E. Gerber ${ }^{132}$ D. J. Hofman, ${ }^{132}$ S. Khalatyan, ${ }^{132}$ F. Lacroix, ${ }^{132}$ C. O'Brien, ${ }^{132}$ C. Silkworth, ${ }^{132}$ D. Strom, ${ }^{132}$ P. Turner, ${ }^{132}$ N. Varelas, ${ }^{132}$ U. Akgun, ${ }^{133}$ E. A. Albayrak, ${ }^{133}$ B. Bilki, ${ }^{133, \text { eee }}$ W. Clarida, ${ }^{133}$ F. Duru, ${ }^{133}$ S. Griffiths, ${ }^{133}$ J.-P. Merlo, ${ }^{133}$ H. Mermerkaya, ${ }^{133, f f f}$ A. Mestvirishvili, ${ }^{133}$ A. Moeller ${ }^{133}$ J. Nachtman, ${ }^{133}$ C. R. Newsom, ${ }^{133}$ E. Norbeck, ${ }^{133}$ Y. Onel, ${ }^{133}$ F. Ozok, ${ }^{133, g g g}$ S. Sen, ${ }^{133}$ P. Tan, ${ }^{133}$ E. Tiras, ${ }^{133}$ J. Wetzel, ${ }^{133}$ T. Yetkin, ${ }^{133}$ K. Yi, ${ }^{133}$ B. A. Barnett, ${ }^{134}$ B. Blumenfeld, ${ }^{134}$ S. Bolognesi, ${ }^{134}$ D. Fehling, ${ }^{134}$ G. Giurgiu, ${ }^{134}$ A. V. Gritsan, ${ }^{134}$ Z. J. Guo, ${ }^{134}$ G. Hu, ${ }^{134}$ P. Maksimovic,,${ }^{134}$ M. Swartz, ${ }^{134}$ A. Whitbeck, ${ }^{134}$ P. Baringer, ${ }^{135}$ A. Bean, ${ }^{135}$

G. Benelli, ${ }^{135}$ R. P. Kenny Iii, ${ }^{135}$ M. Murray, ${ }^{135}$ D. Noonan, ${ }^{135}$ S. Sanders,${ }^{135}$ R. Stringer, ${ }^{135}$ G. Tinti, ${ }^{135}$

J. S. Wood, ${ }^{135}$ A. F. Barfuss, ${ }^{136}$ T. Bolton, ${ }^{136}$ I. Chakaberia, ${ }^{136}$ A. Ivanov, ${ }^{136}$ S. Khalil,${ }^{136}$ M. Makouski, ${ }^{136}$ Y. Maravin, ${ }^{136}$ S. Shrestha, ${ }^{136}$ I. Svintradze, ${ }^{136}$ J. Gronberg,,${ }^{137}$ D. Lange,${ }^{137}$ F. Rebassoo, ${ }^{137}$ D. Wright,,${ }^{137}$ A. Baden, ${ }^{138}$ B. Calvert, ${ }^{138}$ S. C. Eno, ${ }^{138}$ J. A. Gomez, ${ }^{138}$ N. J. Hadley, ${ }^{138}$ R. G. Kellogg, ${ }^{138}$ M. Kirn, ${ }^{138}$ T. Kolberg, ${ }^{138}$ Y. Lu, ${ }^{138}$ M. Marionneau, ${ }^{138}$ A. C. Mignerey, ${ }^{138}$ K. Pedro, ${ }^{138}$ A. Peterman, ${ }^{138}$ A. Skuja, ${ }^{138}$ J. Temple, ${ }^{138}$ M. B. Tonjes, ${ }^{138}$ S. C. Tonwar, ${ }^{138}$ A. Apyan, ${ }^{139}$ G. Bauer, ${ }^{139}$ J. Bendavid, ${ }^{139}$ W. Busza, ${ }^{139}$ E. Butz, ${ }^{139}$ I. A. Cali, ${ }^{139}$ M. Chan, ${ }^{139}$ V. Dutta, ${ }^{139}$ G. Gomez Ceballos, ${ }^{139}$ M. Goncharov, ${ }^{139}$ Y. Kim,,${ }^{139}$ M. Klute, ${ }^{139}$ K. Krajczar, ${ }^{139, \text { hhh }}$ A. Levin, ${ }^{139}$ P. D. Luckey, ${ }^{139}$ T. Ma, ${ }^{139}$ S. Nahn, ${ }^{139}$ C. Paus, ${ }^{139}$ D. Ralph, ${ }^{139}$ C. Roland, ${ }^{139}$ G. Roland, ${ }^{139}$ M. Rudolph, ${ }^{139}$ G. S. F. Stephans, ${ }^{139}$ F. Stöckli, ${ }^{139}$ K. Sumorok, ${ }^{139}$ K. Sung, ${ }^{139}$ D. Velicanu, ${ }^{139}$ E. A. Wenger, ${ }^{139}$ R. Wolf,,${ }^{139}$ B. Wyslouch, ${ }^{139}$ M. Yang, ${ }^{139}$ Y. Yilmaz, ${ }^{139}$ A. S. Yoon, ${ }^{139}$ M. Zanetti, ${ }^{139}$ V. Zhukova, ${ }^{139}$ S. I. Cooper, ${ }^{140}$ B. Dahmes, ${ }^{140}$ A. De Benedetti, ${ }^{140}$ G. Franzoni, ${ }^{140}$ A. Gude,${ }^{140}$ S. C. Kao, ${ }^{140}$ K. Klapoetke,${ }^{140}$

Y. Kubota ${ }^{140}$ J. Mans,${ }^{140}$ N. Pastika,${ }^{140}$ R. Rusack,${ }^{140}$ M. Sasseville, ${ }^{140}$ A. Singovsky, ${ }^{140}$ N. Tambe,${ }^{140}$ J. Turkewitz, ${ }^{140}$ L. M. Cremaldi, ${ }^{141}$ R. Kroeger, ${ }^{141}$ L. Perera, ${ }^{141}$ R. Rahmat,${ }^{141}$ D. A. Sanders, ${ }^{141}$ E. Avdeeva, ${ }^{142}$

K. Bloom,${ }^{142}$ S. Bose,${ }^{142}$ D. R. Claes, ${ }^{142}$ A. Dominguez, ${ }^{142}$ M. Eads,${ }^{142}$ J. Keller, ${ }^{142}$ I. Kravchenko, ${ }^{142}$ J. Lazo-Flores, ${ }^{142}$ S. Malik, ${ }^{142}$ G. R. Snow, ${ }^{142}$ A. Godshalk, ${ }^{143}$ I. Iashvili, ${ }^{143}$ S. Jain, ${ }^{143}$ A. Kharchilava, ${ }^{143}$ A. Kumar, ${ }^{143}$ S. Rappoccio, ${ }^{143}$ G. Alverson, ${ }^{144}$ E. Barberis, ${ }^{144}$ D. Baumgartel, ${ }^{144}$ M. Chasco,${ }^{144}$ J. Haley, ${ }^{144}$ D. Nash, ${ }^{144}$ T. Orimoto, ${ }^{144}$ D. Trocino, ${ }^{144}$ D. Wood, ${ }^{144}$ J. Zhang, ${ }^{144}$ A. Anastassov, ${ }^{145}$ K. A. Hahn, ${ }^{145}$ A. Kubik, ${ }^{145}$ L. Lusito, ${ }^{145}$ N. Mucia, ${ }^{145}$ N. Odell, ${ }^{145}$ R. A. Ofierzynski, ${ }^{145}$ B. Pollack, ${ }^{145}$ A. Pozdnyakov, ${ }^{145}$ M. Schmitt,,${ }^{145}$

S. Stoynev, ${ }^{145}$ M. Velasco, ${ }^{145}$ S. Won, ${ }^{145}$ L. Antonelli, ${ }^{146}$ D. Berry, ${ }^{146}$ A. Brinkerhoff, ${ }^{146}$ K. M. Chan, ${ }^{146}$ M. Hildreth, ${ }^{146}$ C. Jessop, ${ }^{146}$ D. J. Karmgard, ${ }^{146}$ J. Kolb, ${ }^{146}$ K. Lannon, ${ }^{146}$ W. Luo, ${ }^{146}$ S. Lynch, ${ }^{146}$ N. Marinelli, ${ }^{146}$ D. M. Morse, ${ }^{146}$ T. Pearson, ${ }^{146}$ M. Planer, ${ }^{146}$ R. Ruchti, ${ }^{146}$ J. Slaunwhite, ${ }^{146}$ N. Valls, ${ }^{146}$ M. Wayne, ${ }^{146}$ M. Wolf, ${ }^{146}$ B. Bylsma, ${ }^{147}$ L. S. Durkin, ${ }^{147}$ C. Hill, ${ }^{147}$ R. Hughes,${ }^{147}$ K. Kotov,${ }^{147}$ T. Y. Ling,${ }^{147}$ D. Puigh,${ }^{147}$ M. Rodenburg, ${ }^{147}$ C. Vuosalo, ${ }^{147}$ G. Williams, ${ }^{147}$ B. L. Winer, ${ }^{147}$ E. Berry, ${ }^{148}$ P. Elmer ${ }^{148}$ V. Halyo, ${ }^{148}$ P. Hebda, ${ }^{148}$ J. Hegeman, ${ }^{148}$

A. Hunt, ${ }^{148}$ P. Jindal, ${ }^{148}$ S. A. Koay, ${ }^{148}$ D. Lopes Pegna, ${ }^{148}$ P. Lujan, ${ }^{148}$ D. Marlow, ${ }^{148}$ T. Medvedeva,${ }^{148}$

M. Mooney, ${ }^{148}$ J. Olsen, ${ }^{148}$ P. Piroué, ${ }^{148}$ X. Quan, ${ }^{148}$ A. Raval, ${ }^{148}$ H. Saka, ${ }^{148}$ D. Stickland,${ }^{148}$ C. Tully, ${ }^{148}$ J. S. Werner, ${ }^{148}$ A. Zuranski, ${ }^{148}$ E. Brownson, ${ }^{149}$ A. Lopez,${ }^{149}$ H. Mendez, ${ }^{149}$ J. E. Ramirez Vargas, ${ }^{149}$ E. Alagoz, ${ }^{150}$ V.E. Barnes, ${ }^{150}$ D. Benedetti, ${ }^{150}$ G. Bolla, ${ }^{150}$ D. Bortoletto, ${ }^{150}$ M. De Mattia, ${ }^{150}$ A. Everett, ${ }^{150} \mathrm{Z}$. Hu, ${ }^{150}$ M. Jones, ${ }^{150}$ O. Koybasi, ${ }^{150}$ M. Kress, ${ }^{150}$ A. T. Laasanen, ${ }^{150}$ N. Leonardo, ${ }^{150}$ V. Maroussov, ${ }^{150}$ P. Merkel, ${ }^{150}$ D. H. Miller,${ }^{150}$ N. Neumeister ${ }^{150}$ I. Shipsey, ${ }^{150}$ D. Silvers, ${ }^{150}$ A. Svyatkovskiy, ${ }^{150}$ M. Vidal Marono, ${ }^{150}$ H. D. Yoo, ${ }^{150}$ J. Zablocki ${ }^{150}$ Y. Zheng, ${ }^{150}$ S. Guragain ${ }^{151}$ N. Parashar, ${ }^{151}$ A. Adair, ${ }^{152}$ B. Akgun, ${ }^{152}$ C. Boulahouache, ${ }^{152}$ K. M. Ecklund, ${ }^{152}$ F. J. M. Geurts, ${ }^{152}$ W. Li, ${ }^{152}$ B. P. Padley, ${ }^{152}$ R. Redjimi, ${ }^{152}$ J. Roberts, ${ }^{152}$ J. Zabel, ${ }^{152}$ B. Betchart, ${ }^{153}$ A. Bodek, ${ }^{153}$ Y. S. Chung, ${ }^{153}$ R. Covarelli, ${ }^{153}$ P. de Barbaro, ${ }^{153}$ R. Demina, ${ }^{153}$ Y. Eshaq, ${ }^{153}$ T. Ferbel,${ }^{153}$ A. Garcia-Bellido, ${ }^{153}$ P. Goldenzweig, ${ }^{153}$ J. Han, ${ }^{153}$ A. Harel, ${ }^{153}$ D. C. Miner, ${ }^{153}$ D. Vishnevskiy, ${ }^{153}$ M. Zielinski, ${ }^{153}$ A. Bhatti, ${ }^{154}$ R. Ciesielski, ${ }^{154}$ L. Demortier, ${ }^{154}$ K. Goulianos, ${ }^{154}$ G. Lungu, ${ }^{154}$ S. Malik, ${ }^{154}$ C. Mesropian, ${ }^{154}$ S. Arora, ${ }^{155}$ 
A. Barker, ${ }^{155}$ J. P. Chou, ${ }^{155}$ C. Contreras-Campana, ${ }^{155}$ E. Contreras-Campana, ${ }^{155}$ D. Duggan, ${ }^{155}$ D. Ferencek, ${ }^{155}$ Y. Gershtein, ${ }^{155}$ R. Gray, ${ }^{155}$ E. Halkiadakis, ${ }^{155}$ D. Hidas, ${ }^{155}$ A. Lath, ${ }^{155}$ S. Panwalkar, ${ }^{155}$ M. Park,${ }^{155}$ R. Patel,,${ }^{155}$ V. Rekovic, ${ }^{155}$ J. Robles, ${ }^{155}$ K. Rose, ${ }^{155}$ S. Salur, ${ }^{155}$ S. Schnetzer, ${ }^{155}$ C. Seitz, ${ }^{155}$ S. Somalwar, ${ }^{155}$ R. Stone,${ }^{155}$ S. Thomas, ${ }^{155}$ M. Walker, ${ }^{155}$ G. Cerizza, ${ }^{156}$ M. Hollingsworth, ${ }^{156}$ S. Spanier, ${ }^{156}$ Z. C. Yang, ${ }^{156}$ A. York, ${ }^{156}$ R. Eusebi, ${ }^{157}$ W. Flanagan, ${ }^{157}$ J. Gilmore, ${ }^{157}$ T. Kamon,,${ }^{157, \text { iii }}$ V. Khotilovich, ${ }^{157}$ R. Montalvo, ${ }^{157}$ I. Osipenkov, ${ }^{157}$ Y. Pakhotin, ${ }^{157}$ A. Perloff, ${ }^{157}$ J. Roe, ${ }^{157}$ A. Safonov, ${ }^{157}$ T. Sakuma, ${ }^{157}$ S. Sengupta, ${ }^{157}$ I. Suarez, ${ }^{157}$ A. Tatarinov, ${ }^{157}$

D. Toback, ${ }^{157}$ N. Akchurin, ${ }^{158}$ J. Damgov,${ }^{158}$ C. Dragoiu, ${ }^{158}$ P. R. Dudero, ${ }^{158}$ C. Jeong, ${ }^{158}$ K. Kovitanggoon, ${ }^{158}$ S. W. Lee, ${ }^{158}$ T. Libeiro, ${ }^{158}$ I. Volobouev, ${ }^{158}$ E. Appelt, ${ }^{159}$ A. G. Delannoy, ${ }^{159}$ C. Florez, ${ }^{159}$ S. Greene,${ }^{159}$ A. Gurrola, ${ }^{159}$ W. Johns, ${ }^{159}$ P. Kurt, ${ }^{159}$ C. Maguire, ${ }^{159}$ A. Melo, ${ }^{159}$ M. Sharma, ${ }^{159}$ P. Sheldon, ${ }^{159}$ B. Snook,${ }^{159}$ S. Tuo, ${ }^{159}$ J. Velkovska, ${ }^{159}$ M. W. Arenton, ${ }^{160}$ M. Balazs, ${ }^{160}$ S. Boutle, ${ }^{160}$ B. Cox,${ }^{160}$ B. Francis, ${ }^{160}$ J. Goodell, ${ }^{160}$ R. Hirosky, ${ }^{160}$ A. Ledovskoy, ${ }^{160}$ C. Lin,${ }^{160}$ C. Neu, ${ }^{160}$ J. Wood, ${ }^{160}$ S. Gollapinni, ${ }^{161}$ R. Harr, ${ }^{161}$ P.E. Karchin, ${ }^{161}$ C. Kottachchi Kankanamge Don, ${ }^{161}$ P. Lamichhane, ${ }^{161}$ A. Sakharov, ${ }^{161}$ M. Anderson, ${ }^{162}$ D. A. Belknap, ${ }^{162}$ L. Borrello, ${ }^{162}$ D. Carlsmith, ${ }^{162}$ M. Cepeda, ${ }^{162}$ S. Dasu, ${ }^{162}$ E. Friis, ${ }^{162}$ L. Gray, ${ }^{162}$ K. S. Grogg, ${ }^{162}$ M. Grothe, ${ }^{162}$ R. Hall-Wilton, ${ }^{162}$ M. Herndon, ${ }^{162}$ A. Hervé, ${ }^{162}$ P. Klabbers, ${ }^{162}$ J. Klukas, ${ }^{162}$ A. Lanaro, ${ }^{162}$ C. Lazaridis, ${ }^{162}$ R. Loveless, ${ }^{162}$ A. Mohapatra, ${ }^{162}$ I. Ojalvo, ${ }^{162}$ F. Palmonari, ${ }^{162}$ G. A. Pierro, ${ }^{162}$ I. Ross, ${ }^{162}$ A. Savin, ${ }^{162}$ W. H. Smith, ${ }^{162}$ and J. Swanson ${ }^{162}$

(CMS Collaboration)

\author{
${ }^{1}$ Yerevan Physics Institute, Yerevan, Armenia \\ ${ }^{2}$ Institut für Hochenergiephysik der OeAW, Wien, Austria \\ ${ }^{3}$ National Centre for Particle and High Energy Physics, Minsk, Belarus \\ ${ }^{4}$ Universiteit Antwerpen, Antwerpen, Belgium \\ ${ }^{5}$ Vrije Universiteit Brussel, Brussel, Belgium \\ ${ }^{6}$ Université Libre de Bruxelles, Bruxelles, Belgium \\ ${ }^{7}$ Ghent University, Ghent, Belgium \\ ${ }^{8}$ Université Catholique de Louvain, Louvain-la-Neuve, Belgium \\ ${ }^{9}$ Université de Mons, Mons, Belgium \\ ${ }^{10}$ Centro Brasileiro de Pesquisas Fisicas, Rio de Janeiro, Brazil \\ ${ }^{11}$ Universidade do Estado do Rio de Janeiro, Rio de Janeiro, Brazil \\ ${ }^{12 a}$ Universidade Estadual Paulista, São Paulo, Brazil \\ ${ }^{12 \mathrm{~b}}$ Universidade Federal do ABC, São Paulo, Brazil \\ ${ }^{13}$ Institute for Nuclear Research and Nuclear Energy, Sofia, Bulgaria \\ ${ }^{14}$ University of Sofia, Sofia, Bulgaria \\ ${ }^{15}$ Institute of High Energy Physics, Beijing, China \\ ${ }^{16}$ State Key Lab. of Nucl. Phys. and Tech., Peking University, Beijing, China \\ ${ }^{17}$ Universidad de Los Andes, Bogota, Colombia \\ ${ }^{18}$ Technical University of Split, Split, Croatia \\ ${ }^{19}$ University of Split, Split, Croatia \\ ${ }^{20}$ Institute Rudjer Boskovic, Zagreb, Croatia \\ ${ }^{21}$ University of Cyprus, Nicosia, Cyprus \\ ${ }^{22}$ Charles University, Prague, Czech Republic \\ ${ }^{23}$ Academy of Scientific Research and Technology of the Arab Republic of Egypt, \\ Egyptian Network of High Energy Physics, Cairo, Egypt \\ ${ }^{24}$ National Institute of Chemical Physics and Biophysics, Tallinn, Estonia \\ ${ }^{25}$ Department of Physics, University of Helsinki, Helsinki, Finland \\ ${ }^{26}$ Helsinki Institute of Physics, Helsinki, Finland \\ ${ }^{27}$ Lappeenranta University of Technology, Lappeenranta, Finland \\ ${ }^{28}$ DSM/IRFU, CEA/Saclay, Gif-sur-Yvette, France \\ ${ }^{29}$ Laboratoire Leprince-Ringuet, Ecole Polytechnique, IN2P3-CNRS, Palaiseau, France \\ ${ }^{30}$ Institut Pluridisciplinaire Hubert Curien, Université de Strasbourg, \\ Université de Haute Alsace Mulhouse, CNRS/IN2P3, Strasbourg, France \\ ${ }^{31}$ Centre de Calcul de l'Institut National de Physique Nucleaire et de Physique des Particules, CNRS/IN2P3, Villeurbanne, France \\ ${ }^{32}$ Université de Lyon, Université Claude Bernard Lyon 1, CNRS-IN2P3, Institut de Physique Nucléaire de Lyon, Villeurbanne, France \\ ${ }^{33}$ Institute of High Energy Physics and Informatization, Tbilisi State University, Tbilisi, Georgia \\ ${ }^{34}$ RWTH Aachen University, I. Physikalisches Institut, Aachen, Germany \\ ${ }^{35}$ RWTH Aachen University, III. Physikalisches Institut A, Aachen, Germany
}


${ }^{36}$ RWTH Aachen University, III. Physikalisches Institut B, Aachen, Germany

${ }^{37}$ Deutsches Elektronen-Synchrotron, Hamburg, Germany

${ }^{38}$ University of Hamburg, Hamburg, Germany

${ }^{39}$ Institut für Experimentelle Kernphysik, Karlsruhe, Germany

${ }^{40}$ Institute of Nuclear Physics “Demokritos,” Aghia Paraskevi, Greece

${ }^{41}$ University of Athens, Athens, Greece

${ }^{42}$ University of Ioánnina, Ioánnina, Greece

${ }^{43}$ KFKI Research Institute for Particle and Nuclear Physics, Budapest, Hungary

${ }^{44}$ Institute of Nuclear Research ATOMKI, Debrecen, Hungary

${ }^{45}$ University of Debrecen, Debrecen, Hungary

${ }^{46}$ Panjab University, Chandigarh, India

${ }^{47}$ University of Delhi, Delhi, India

${ }^{48}$ Saha Institute of Nuclear Physics, Kolkata, India

${ }^{49}$ Bhabha Atomic Research Centre, Mumbai, India

${ }^{50}$ Tata Institute of Fundamental Research-EHEP, Mumbai, India

${ }^{51}$ Tata Institute of Fundamental Research-HECR, Mumbai, India

${ }^{52}$ Institute for Research in Fundamental Sciences (IPM), Tehran, Iran

${ }^{53 a}$ INFN Sezione di Bari, Bari, Italy

${ }^{53 \mathrm{~b}}$ Università di Bari, Bari, Italy

${ }^{53 \mathrm{c}}$ Politecnico di Bari, Bari, Italy

${ }^{54 a}$ INFN Sezione di Bologna, Bologna, Italy

${ }^{54 b}$ Università di Bologna, Bologna, Italy

${ }^{55}$ aNFN Sezione di Catania, Catania, Italy

${ }^{55 \mathrm{~b}}$ Università di Catania, Catania, Italy

${ }^{56 a}$ INFN Sezione di Firenze, Firenze, Italy

${ }^{56 \mathrm{~b}}$ Università di Firenze, Firenze, Italy

${ }^{57}$ INFN Laboratori Nazionali di Frascati, Frascati, Italy

${ }^{58 \mathrm{a}}$ INFN Sezione di Genova, Genova, Italy

${ }^{58 \mathrm{~b}}$ Università di Genova, Genova, Italy

${ }^{59 a}$ INFN Sezione di Milano-Bicocca, Milano, Italy

${ }^{59 \mathrm{~b}}$ Università di Milano-Bicocca, Milano, Italy

${ }^{60 a}$ INFN Sezione di Napoli, Napoli, Italy

${ }^{60 \mathrm{~b}}$ Università di Napoli 'Federico II', Napoli, Italy

${ }^{60 c}$ Università della Basilicata (Potenza), Napoli, Italy

${ }^{60 \mathrm{~d}}$ Università G. Marconi (Roma), Napoli, Italy

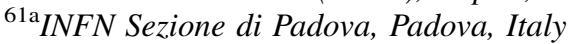

${ }^{61 \mathrm{~b}}$ Università di Padova, Padova, Italy

${ }^{61 \mathrm{c}}$ Università di Trento (Trento), Padova, Italy

${ }^{62 a}$ INFN Sezione di Pavia, Pavia, Italy ${ }^{62 \mathrm{~b}}$ Università di Pavia, Pavia, Italy

${ }^{63 a}$ INFN Sezione di Perugia, Perugia, Italy

${ }^{63 \mathrm{~b}}$ Università di Perugia, Perugia, Italy

${ }^{64 \mathrm{a}}$ INFN Sezione di Pisa, Pisa, Italy

${ }^{64 \mathrm{~b}}$ Università di Pisa, Pisa, Italy

${ }^{64 \mathrm{c}}$ Scuola Normale Superiore di Pisa, Pisa, Italy

${ }^{65 a}$ INFN Sezione di Roma, Roma, Italy

${ }^{65 \mathrm{~b}}$ Università di Roma, Roma, Italy

${ }^{66 \mathrm{a}}$ INFN Sezione di Torino, Torino, Italy

${ }^{66 \mathrm{~b}}$ Università di Torino, Torino, Italy

${ }^{66 \mathrm{c}}$ Università del Piemonte Orientale (Novara), Torino, Italy

${ }^{67 \mathrm{a} I N F N}$ Sezione di Trieste, Trieste, Italy

${ }^{67 \mathrm{~b}}$ Università di Trieste, Trieste, Italy

${ }^{68}$ Kangwon National University, Chunchon, Korea

${ }^{69}$ Kyungpook National University, Daegu, Korea

${ }^{70}$ Chonnam National University, Institute for Universe and Elementary Particles, Kwangju, Korea

${ }^{71}$ Korea University, Seoul, Korea

${ }^{72}$ University of Seoul, Seoul, Korea

${ }^{73}$ Sungkyunkwan University, Suwon, Korea

${ }^{74}$ Vilnius University, Vilnius, Lithuania

${ }^{75}$ Centro de Investigacion y de Estudios Avanzados del IPN, Mexico City, Mexico

${ }^{76}$ Universidad Iberoamericana, Mexico City, Mexico 
${ }^{77}$ Benemerita Universidad Autonoma de Puebla, Puebla, Mexico

${ }^{78}$ Universidad Autónoma de San Luis Potosí, San Luis Potosí, Mexico

${ }^{79}$ University of Auckland, Auckland, New Zealand

${ }^{80}$ University of Canterbury, Christchurch, New Zealand

${ }^{81}$ National Centre for Physics, Quaid-I-Azam University, Islamabad, Pakistan

${ }^{82}$ National Centre for Nuclear Research, Swierk, Poland

${ }^{83}$ Institute of Experimental Physics, Faculty of Physics, University of Warsaw, Warsaw, Poland

${ }^{84}$ Laboratório de Instrumentação e Física Experimental de Partículas, Lisboa, Portugal

${ }^{85}$ Joint Institute for Nuclear Research, Dubna, Russia

${ }^{86}$ Petersburg Nuclear Physics Institute, Gatchina (St. Petersburg), Russia

${ }^{87}$ Institute for Nuclear Research, Moscow, Russia

${ }^{88}$ Institute for Theoretical and Experimental Physics, Moscow, Russia

${ }^{89}$ Moscow State University, Moscow, Russia

${ }^{90}$ P.N. Lebedev Physical Institute, Moscow, Russia

${ }^{91}$ State Research Center of Russian Federation, Institute for High Energy Physics, Protvino, Russia

${ }^{92}$ University of Belgrade, Faculty of Physics and Vinca Institute of Nuclear Sciences, Belgrade, Serbia

${ }^{93}$ Centro de Investigaciones Energéticas Medioambientales y Tecnológicas (CIEMAT), Madrid, Spain

${ }^{94}$ Universidad Autónoma de Madrid, Madrid, Spain

${ }^{95}$ Universidad de Oviedo, Oviedo, Spain

${ }^{96}$ Instituto de Física de Cantabria (IFCA), CSIC-Universidad de Cantabria, Santander, Spain

${ }^{97}$ CERN, European Organization for Nuclear Research, Geneva, Switzerland

${ }^{98}$ Paul Scherrer Institut, Villigen, Switzerland

${ }^{99}$ Institute for Particle Physics, ETH Zurich, Zurich, Switzerland

${ }^{100}$ Universität Zürich, Zurich, Switzerland

${ }^{101}$ National Central University, Chung-Li, Taiwan

${ }^{102}$ National Taiwan University (NTU), Taipei, Taiwan

${ }^{103}$ Chulalongkorn University, Bangkok, Thailand

${ }^{104}$ Cukurova University, Adana, Turkey

${ }^{105}$ Middle East Technical University, Physics Department, Ankara, Turkey

${ }^{106}$ Bogazici University, Istanbul, Turkey

${ }^{107}$ Istanbul Technical University, Istanbul, Turkey

${ }^{108}$ National Scientific Center, Kharkov Institute of Physics and Technology, Kharkov, Ukraine

${ }^{109}$ University of Bristol, Bristol, United Kingdom

${ }^{110}$ Rutherford Appleton Laboratory, Didcot, United Kingdom

${ }^{111}$ Imperial College, London, United Kingdom

${ }^{112}$ Brunel University, Uxbridge, United Kingdom

${ }^{113}$ Baylor University, Waco, Texas, USA

${ }^{114}$ The University of Alabama, Tuscaloosa, Alabama, USA

${ }^{115}$ Boston University, Boston, Massachusetts, USA

${ }^{116}$ Brown University, Providence, Rhode Island, USA

${ }^{117}$ University of California, Davis, Davis, California, USA

${ }^{118}$ University of California, Los Angeles, California, USA

${ }^{119}$ University of California, Riverside, Riverside, California, USA

${ }^{120}$ University of California, San Diego, La Jolla, California, USA

${ }^{121}$ University of California, Santa Barbara, Santa Barbara, California, USA

${ }^{122}$ California Institute of Technology, Pasadena, California, USA

${ }^{123}$ Carnegie Mellon University, Pittsburgh, Pennsylvaia, USA

${ }^{124}$ University of Colorado at Boulder, Boulder, Colorado, USA

${ }^{125}$ Cornell University, Ithaca, New York, USA

${ }^{126}$ Fairfield University, Fairfield, Connecticut, USA

${ }^{127}$ Fermi National Accelerator Laboratory, Batavia, Illinois, USA

${ }^{128}$ University of Florida, Gainesville, Florida, USA

${ }^{129}$ Florida International University, Miami, Florida, USA

${ }^{130}$ Florida State University, Tallahassee, Florida, USA

${ }^{131}$ Florida Institute of Technology, Melbourne, Florida, USA

${ }^{132}$ University of Illinois at Chicago (UIC), Chicago, Illinois, USA

${ }^{133}$ The University of Iowa, Iowa City, Iowa, USA

${ }^{134}$ Johns Hopkins University, Baltimore, Maryland, USA

${ }^{135}$ The University of Kansas, Lawrence, Kansas, USA

${ }^{136}$ Kansas State University, Manhattan, Kansas, USA

${ }^{137}$ Lawrence Livermore National Laboratory, Livermore, California, USA 


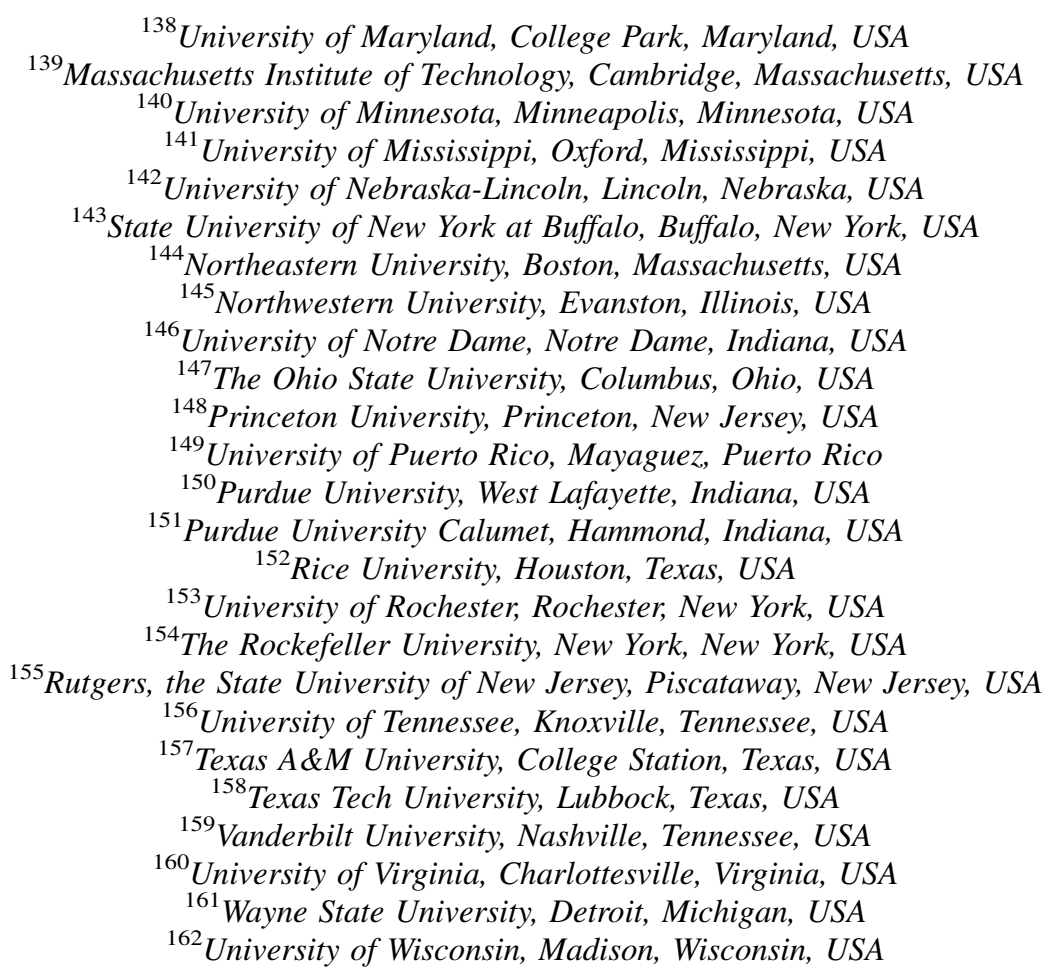

${ }^{\mathrm{a}}$ Deceased.

${ }^{\mathrm{b}}$ Also at Vienna University of Technology, Vienna, Austria.

${ }^{\mathrm{c}}$ Also at CERN, European Organization for Nuclear Research, Geneva, Switzerland.

${ }^{\mathrm{d}}$ Also at National Institute of Chemical Physics and Biophysics, Tallinn, Estonia.

${ }^{\mathrm{e}}$ Also at California Institute of Technology, Pasadena, CA, USA.

${ }^{\mathrm{f}}$ Also at Laboratoire Leprince-Ringuet, Ecole Polytechnique, IN2P3-CNRS, Palaiseau, France.

${ }^{\mathrm{g}}$ Also at Suez Canal University, Suez, Egypt.

${ }^{\mathrm{h}}$ Also at Zewail City of Science and Technology, Zewail, Egypt.

${ }^{\mathrm{i}}$ Also at Cairo University, Cairo, Egypt.

${ }^{\mathrm{j}}$ Also at Fayoum University, El-Fayoum, Egypt.

${ }^{\mathrm{k}}$ Also at Helwan University, Cairo, Egypt.

${ }^{1}$ Also at British University in Egypt, Cairo, Egypt.

${ }^{\mathrm{m}}$ Now at Ain Shams University, Cairo, Egypt.

${ }^{\mathrm{n}}$ Also at National Centre for Nuclear Research, Swierk, Poland.

${ }^{\circ}$ Also at Université de Haute-Alsace, Mulhouse, France.

${ }^{\mathrm{p}}$ Also at Joint Institute for Nuclear Research, Dubna, Russia.

${ }^{\mathrm{q}}$ Also at Moscow State University, Moscow, Russia.

${ }^{\mathrm{r}}$ Also at Brandenburg University of Technology, Cottbus, Germany.

${ }^{\mathrm{s}}$ Also at The University of Kansas, Lawrence, KS, USA.

${ }^{\mathrm{t}}$ Also at Institute of Nuclear Research ATOMKI, Debrecen, Hungary.

${ }^{\text {u} A l s o ~ a t ~ E o ̈ t v o ̈ s ~ L o r a ́ n d ~ U n i v e r s i t y, ~ B u d a p e s t, ~ H u n g a r y . ~}$

${ }^{v}$ Also at Tata Institute of Fundamental Research - HECR, Mumbai, India.

${ }^{\mathrm{w}}$ Now at King Abdulaziz University, Jeddah, Saudi Arabia.

${ }^{\mathrm{x}}$ Also at University of Visva-Bharati, Santiniketan, India.

${ }^{y}$ Also at Sharif University of Technology, Tehran, Iran.

${ }^{\mathrm{z}}$ Also at Isfahan University of Technology, Isfahan, Iran.

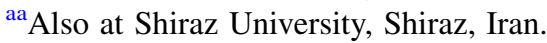

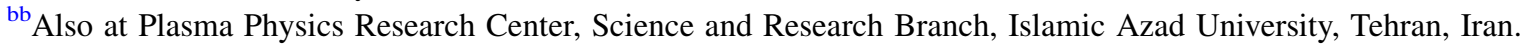

${ }^{\mathrm{cc}}$ Also at Facoltà Ingegneria, Università di Roma, Roma, Italy.

${ }^{\mathrm{dd}}$ Also at Università degli Studi Guglielmo Marconi, Roma, Italy.

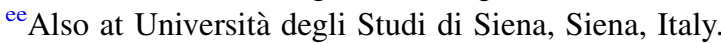

${ }^{\mathrm{ff}}$ Also at University of Bucharest, Faculty of Physics, Bucuresti-Magurele, Romania. 
${ }^{g g}$ Also at Faculty of Physics of University of Belgrade, Belgrade, Serbia.

${ }^{\text {hh }}$ Also at University of California, Los Angeles, CA, USA.

${ }^{i i}$ Also at Scuola Normale e Sezione dell'INFN, Pisa, Italy.

${ }^{\mathrm{jj}}$ Also at INFN Sezione di Roma, Roma, Italy.

${ }^{\mathrm{kk}}$ Also at University of Athens, Athens, Greece.

${ }^{11}$ Also at Rutherford Appleton Laboratory, Didcot, United Kingdom.

${ }^{\mathrm{mm}}$ Also at Paul Scherrer Institut, Villigen, Switzerland.

${ }^{\mathrm{nn}}$ Also at Institute for Theoretical and Experimental Physics, Moscow, Russia.

${ }^{\circ o}$ Also at Albert Einstein Center for Fundamental Physics, Bern, Switzerland.

${ }^{\mathrm{pp}}$ Also at Gaziosmanpasa University, Tokat, Turkey.

${ }^{\mathrm{qq}}$ Also at Adiyaman University, Adiyaman, Turkey.

${ }^{\mathrm{rr}}$ Also at Izmir Institute of Technology, Izmir, Turkey.

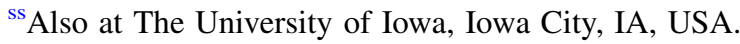

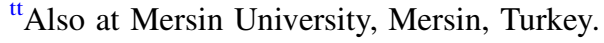

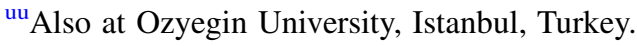

${ }^{\mathrm{vv}}$ Also at Kafkas University, Kars, Turkey.

${ }^{\text {ww }}$ Also at Suleyman Demirel University, Isparta, Turkey.

${ }^{\mathrm{xx}}$ Also at Ege University, Izmir, Turkey.

${ }^{y y}$ Also at School of Physics and Astronomy, University of Southampton, Southampton, United Kingdom.

${ }^{z z}$ Also at INFN Sezione di Perugia, Università di Perugia, Perugia, Italy.

${ }^{\text {aaa }}$ Also at Utah Valley University, Orem, UT, USA.

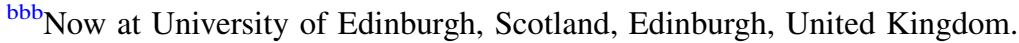

${ }^{c c c}$ Also at Institute for Nuclear Research, Moscow, Russia.

${ }^{\text {ddd }}$ Also at University of Belgrade, Faculty of Physics and Vinca Institute of Nuclear Sciences, Belgrade, Serbia.

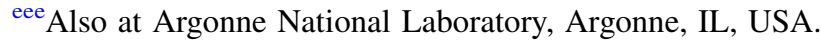

${ }^{f f f}$ Also at Erzincan University, Erzincan, Turkey.

${ }^{\mathrm{ggg}}$ Also at Mimar Sinan University, Istanbul, Istanbul, Turkey.

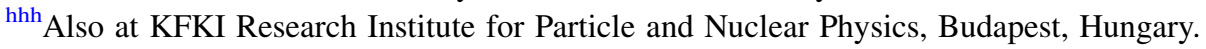

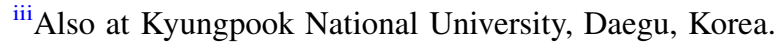

